Pacific

Journal of

Mathematics

SEMICONTINUITY OF AUTOMORPHISM GROUPS OF STRONGLY PSEUDOCONVEX DOMAINS:

THE LOW DIFFERENTIABILITY CASE

Robert E. Greene, KANG-TAE Kim,

Steven G. Krantz and Aeryeong Seo 


\title{
SEMICONTINUITY OF AUTOMORPHISM GROUPS OF STRONGLY PSEUDOCONVEX DOMAINS: THE LOW DIFFERENTIABILITY CASE
}

\author{
Robert E. Greene, Kang-Tae Kim, \\ Steven G. Krantz and Aeryeong Seo
}

\begin{abstract}
We study the semicontinuity of automorphism groups for perturbations of domains in complex space or in complex manifolds. We provide a new approach to the study of such results for domains having minimal boundary smoothness. The emphasis in this study is on the low differentiability assumption and the new methodology developed accordingly.
\end{abstract}

1. Introduction 365

2. Normal families and general semicontinuity of groups of mappings

3. Bergman metric and curvature with $C^{2}$ stability near the strongly pseudoconvex boundary

4. Stable $C^{k}$-extension of automorphisms

5. Conjugation by diffeomorphism 388

6. Concluding remarks

References

\section{Introduction}

It is a familiar perception of everyday life that symmetry is hard to create, but easy to destroy. To make the crooked straight requires some definite effort, but the slightest change can suffice to make the straight a little crooked and hence not straight at all. This perception is easily substantiated in precise form for geometric objects in Euclidean space. It is natural to ask if something similar might apply for automorphism groups in complex analysis, that is, for the group of biholomorphic self-maps of, say, a bounded domain in complex Euclidean space.

In one complex variable, this idea does not yield much, at least in the topologically trivial case. Since all bounded domains that are topologically equivalent to the unit

The second and fourth authors are supported in part by grant 2011-007831 and SRC grant 20110030044 (Center for Geometry and its Applications) of the National Research Foundation of Korea. MSC2010: 32M05.

Keywords: automorphism group, Bergman metric, curvature, extension of holomorphic maps. 
disc are biholomorphic to the unit disc (Riemann mapping theorem, of course), there is not much interest in discussing how the automorphism group varies with the domain: it does not vary at all.

But, in higher dimensions, the idea comes into its own. Domains near the unit ball can have no automorphisms whatever except the identity, and indeed domains with trivial automorphism group are dense in the set of $C^{\infty}$ strongly pseudoconvex domains in the $C^{\infty}$ topology (see [Greene and Krantz 1982a] for detailed references to the literature): the proof of this result in fact goes back really to Poincaré, in effect, since it depends essentially only on counting parameters rather than on the details of local invariant theory, at least once one knows that biholomorphic maps extend smoothly to the boundary [Fefferman 1974]. It is also the case that domains near the unit ball have automorphism groups which are isomorphic to a subgroup of the automorphism group of the ball. Indeed, if a domain is $C^{\infty}$ close enough to the ball, then the domain is either biholomorphic to the ball or its automorphism group is isomorphic to a (closed) subgroup of the unitary group [Greene and Krantz 1982a].

This kind of semicontinuity holds in greater generality [ibid.]. If a $C^{\infty}$ strongly pseudoconvex domain is not biholomorphic to the ball, then there is a neighborhood of the domain in the $C^{\infty}$ topology on the set of all $C^{\infty}$ bounded domains with the property that the automorphism group of every domain in the neighborhood is isomorphic to a subgroup of the automorphism group of the original domain. (The case of the fixed domain being biholomorphic to the ball is as in the previous paragraph.)

The goal of this paper is to explore the possibility of reducing the level of differentiability required for this type of semicontinuity result, both for the fixed domain itself and for the perturbed domains and the topology upon them. We shall show in fact that $C^{\infty}$ can be reduced to $C^{2}$. This is optimal in the sense that $C^{2}$ is the natural setting for the discussion of strong pseudoconvexity and is the lowest level of regularity for which the definition is naturally given. (One can, of course, construct somewhat more intricate and to some extent artificial ideas of strong pseudoconvexity wherein the boundary need not have that much regularity, but these will not be explored here.)

It will turn out that the complex analysis results just discussed can in fact best be treated by changing the whole context to manifolds and general group actions. The role of complex analysis becomes simply to guarantee a kind of uniform compactness discussed in Section 2 in detail.

To put this matter in perspective, it is desirable to recall in outline how the semicontinuity results in [Greene and Krantz 1982a] were obtained. The starting point is the use of normal family arguments. In this context, the setup is as follows. Fix a bounded domain $\Omega_{0}$. Then a sequence of bounded domains $\Omega_{j}$ is considered 
to converge to $\Omega_{0}$ if there is a sequence of maps $\Phi_{j}: \Omega_{0} \rightarrow \Omega_{j}$ which converges to the identity in some appropriate topology. Now, in this situation, a sequence of automorphisms $f_{j}: \Omega_{j} \rightarrow \Omega_{j}$ always has a subsequence $f_{j_{k}}$ such that the maps $\Phi_{j_{k}}^{-1} \circ f_{j_{k}} \circ \Phi_{j_{k}}$ converge to some map of $\Omega_{0}$ to the closure of $\Omega_{0}$. Here convergence means uniform convergence on compact subsets of $\Omega_{0}$.

However, it is relatively easy to show, and it is in fact a classical result that, if the limit mapping is in fact interior, i.e., if its image lies in $\Omega_{0}$ itself, then that limit is an automorphism of $\Omega_{0}$. (A detailed proof is given in [Krantz 2001].) Thus, in trying to relate the automorphisms of the $\Omega_{j}$ 's to those of $\Omega_{0}$, one is interested in situations where it is guaranteed that the family of maps of the sort described always has "nondegenerate" limits; that is, the limits are necessarily the maps into $\Omega_{0}$ itself, with no boundary points in the image.

As it happens, every strongly pseudoconvex bounded domain that is not biholomorphic to the ball has a compact automorphism group. This was proved in [Wong 1977] and has been much generalized since, to the point where the result is not only valid for $C^{2}$ domains but is localized completely: if a sequence of automorphisms has the property that, for some interior point the sequence of the images of the point converge to a $C^{2}$ strongly pseudoconvex boundary point of a domain in a general complex manifold, then the domain is biholomorphic to the ball [Efimov 1995; Gaussier et al. 2002]. This line of thought makes it natural to consider the whole normal families situation for bounded strongly pseudoconvex domains that are not biholomorphic to the ball, which will indeed be the main topic in this paper. However, certain aspects of the situation can be treated with no pseudoconvexity invoked at all. If one simply assumes the relevant kind of nondegeneracy of normal families as a hypothesis, then a semicontinuity result already follows. This matter is treated in Section 2.

It is natural to ask when that nondegeneracy hypothesis is satisfied, that is, under what conditions of a more familiar sort the nondegeneracy condition (stably interior) that is required in Section 2 is sure to hold. As we shall see, it in fact always holds under the hypothesis of $C^{2}$ strong pseudoconvexity of the boundary of $\Omega_{0}\left(\Omega_{0}\right.$ not biholomorphic to the ball) and the assumption that the $\Omega_{j}$ converge to $\Omega_{0}$ in the $C^{2}$ topology. How this arises requires some explanation.

The semicontinuity of automorphism groups in the $C^{2}$ case will be obtained in this paper again by using curvature invariants to bound the distance of orbits from the boundary stably. But the stability of the asymptotic constancy of holomorphic curvature of the Bergman metric will be obtained without using the Fefferman expansion, thus avoiding the need for a large number of derivatives. Instead, the behavior of the holomorphic sectional curvature of the Bergman metric will be analyzed using the "scaling method," as explained in Section 3. The possibility of using the scaling method depends on noting that the holomorphic sectional 
curvature can be expressed in terms of a special basis for the Hilbert space of square integrable holomorphic functions (see [Greene and Wu 1979] and [Epstein 1965] for the special basis concept in generality). This means that one can detour around the rather awkward formulas from Riemannian geometry that express the curvature tensor as a whole in terms of the metric and operate instead with more directly accessible aspects of the fundamental Bergman construction.

In the last section of the present paper, a more refined kind of semicontinuity result involving not just isomorphism to a subgroup but isomorphism to a subgroup via diffeomorphism conjugacy will be obtained for strongly pseudoconvex domains with low boundary regularity. For technical reasons, the regularity cannot be quite reduced to the $C^{2}$ level which would be all that is needed for the subgroup semicontinuity. This is related to the present Theorem 4.3, a uniform version of Lempert's extension theorem for biholomorphic mappings of bounded domains with $C^{k, \alpha}$ smooth boundaries for every $k \geq 2$. For the conjugacy arguments, the necessary regularity here turns out to be $C^{4, \alpha}$. It may be possible that diffeomorphism conjugacy also applies in the $C^{2}$ case, but this result cannot be proved by the methods used here.

It is worth noting that in [Greene and Krantz 1985] we established a version of the semicontinuity theorem for automorphism groups in the context of $C^{2}$ convergence. That paper was an important first step in the program we are developing here. The role of holomorphic curvature of the Bergman metric was replaced by the quotient invariant, that is the Carathéodory volume divided by the Kobayashi-Eisenmann volume. But the curvature methods here are of independent interest, and the needed stable uniformity of $C^{k}$ extension of automorphisms, for low $k$, is checked here in Theorem 4.3.

\section{Normal families and general semicontinuity of groups of mappings}

In this section, some very general results will be discussed about groups of diffeomorphisms of open sets in Euclidean spaces. The fundamental idea is that, as far as semicontinuity of the groups is concerned, the noncompact case can be converted to the compact case. This is, more precisely, true as far as semicontinuity in the sense of isomorphism to a subgroup is concerned. We begin with a definition of an appropriate idea of convergence of the open sets. For convenience, and without any particular loss of generality, we restrict our attention to connected open sets, i.e., domains.

Definition 2.1. A sequence $\Omega_{j}$ of connected open sets, or domains, in a Euclidean space $\mathbb{R}^{n}$, is said to containment-converge to a limit domain $\Omega_{0}$ if, for every compact subset $K$ of $\Omega_{0}, K$ is contained in $\Omega_{j}$ for all sufficiently large $j$.

Definition 2.2. If the sequence $\left\{\Omega_{j}\right\}$ of domains containment-converges to a domain $\Omega_{0}$, then a sequence of $C^{\infty}$ mappings $f_{j}: \Omega_{j} \rightarrow \mathbb{R}^{n}$ is said to converge $C^{\infty}$ 
normally if, for each compact subset $K$ of $\Omega_{0}$, the mappings $f_{j}$ and their derivatives of all orders converge uniformly on $K$.

Note here that the $f_{j}$ are defined in a neighborhood of $K$, any compact set $K$, for all $j$ sufficiently large, so that the desired uniform convergence indeed makes sense.

For our next definition, we recall that there is a metric, to be denoted $\gamma_{K}$, on the set of all $C^{\infty}$ mappings of a neighborhood of a compact subset $K$ to $\mathbb{R}^{n}$ such that convergence in this metric is equivalent to convergence of the mappings and their derivatives of all orders uniformly on the compact set $K$ (see [Greene and Krantz 1982b], for example).

Definition 2.3. Suppose that $\left\{\Omega_{j}\right\}$ is a sequence of domains which containmentconverges to a domain $\Omega_{0}$ and also suppose that, for each $j, G_{j}$ is a group of diffeomorphisms of $\Omega_{j}$, and that $G_{0}$ is a group of diffeomorphisms of $\Omega_{0}$. We say that the sequence of groups $G_{j}$ converges normally to $G_{0}$ if, for each compact subset $K$ of $\Omega_{0}$ and for each $\epsilon>0$, there is a $j_{\epsilon, K}$ such that, for each $j>j_{\epsilon, K}$ and each $\phi_{j} \in G_{j}$, the mapping $\left.\phi_{j}\right|_{K}$ lies within $\gamma_{K}$-distance $\epsilon$ of some element of $G_{0}$.

In case one has not domains, but compact manifolds and compact groups, then the situation is as follows:

Lemma 2.4 (from [Ebin 1968]; cf. [Kim 1987] and [Greene et al. 2011]). If $M$ is a compact manifold and if $G_{j}$ is a sequence of compact subgroups of the diffeomorphism group of $M$ [in the topology determined by the metric $\gamma_{M}$ ] such that $G_{j}$ converges to the compact subgroup $G_{0}$ then, for all $j$ sufficiently large, $G_{j}$ is isomorphic to a subgroup of $G_{0}$. Moreover, the isomorphism can be obtained by conjugation by a diffeomorphism $\phi_{j}$ and the $\phi_{j}$ can be chosen to converge to the identity [again in the topology determined by the metric $\gamma_{M}$ ].

This follows from the original result of [Ebin 1968] as follows. Averaging on arbitrary Riemannian metric on $M$ with respect to the action of $G$ gives a Riemannian metric $g$ on $M$ which is $G$-invariant; i.e., $G \subset \operatorname{Isom}(g)$, where $\operatorname{Isom}(g)$ is the isometry group of $g$. Averaging $g$ with respect to the action of $G_{j}$ for each $j$ yields Riemannian metric $g_{j}$ with $G_{j} \subset \operatorname{Isom}\left(g_{j}\right)$ for each $j$. Since the elements of $G_{j}$ are close to the elements of $G$ by hypothesis, when $j$ is large, it follows easily that the sequence $\left\{g_{j}\right\}$ of metrics converges $C^{\infty}$ to $g$. By Ebin's original result, there are, for all $j$ sufficiently large, diffeomorphisms $\phi_{j}: M \rightarrow M$ such that $\phi_{j}$ conjugates $\operatorname{Isom}\left(g_{j}\right)$ to a subgroup $\phi_{j} \circ \beta \circ \phi_{j}^{-1} \in \operatorname{Isom}(g)$ for all $\beta \in \operatorname{Isom}\left(g_{j}\right)$ of $\operatorname{Isom}(g)$; moreover, the sequence $\left\{\phi_{j}\right\}$ can be taken to converge $C^{\infty}$ to the identity. Then each $\phi_{j}$ conjugates $G_{j}$ (for $j$ large) to a subgroup $\widehat{G}_{j}$ of $\operatorname{Isom}(g)$, with $\widehat{G}_{j}$ $C^{\infty}$-close to $G$. By a classical theorem of [Montgomery and Samelson 1943], it follows that $\widehat{G}_{j}$ is isomorphic to a subgroup of $G$ via conjugation by an element $\sigma_{j}$ of $\operatorname{Isom}(g)$, with the $\sigma_{j}$ converging to the identity. 
This point will arise again in a slightly different form in Section 5, q.v.

Our goal here is to show how to reduce the domain case to the compact manifold situation described in Lemma 2.4. Specifically, we want to prove the following proposition:

Proposition 2.5. Suppose that $\left\{\Omega_{j}\right\}$ is a sequence of bounded domains in $\mathbb{R}^{N}$ which containment-converges to $\Omega_{0}$ in the sense of Definition 2.1 and that, for each $j, G_{j}$ is a compact group of diffeomorphisms of $\mathrm{cl}\left(\Omega_{j}\right)$ and that the sequence $\left\{G_{j}\right\}$ converges $C^{\infty}$ normally to a compact group $G_{0}$ of diffeomorphisms of $\operatorname{cl}\left(\Omega_{0}\right)$ [convergence in the sense of Definition 2.3]. Here, of course, cl denotes the closure of the indicated set. Then, for all sufficiently large $j$, the group $G_{j}$ is isomorphic to a subgroup of $G_{0}$.

Proof. The essential tool is to use group-invariant exhaustion functions to find a smoothly bounded subdomain of $\Omega_{0}$ that is taken to itself by each element of the group $G_{0}$ and then to pass to the "double" of these subdomains to form a compact manifold. Then one does a similar construction to nearby $G_{j}$-invariant subdomains of $\Omega_{j}$ and thus attains the situation of Ebin's theorem. We now describe this situation in more detail, following the arguments developed in [Greene and Krantz 1982b]:

Definition 2.6. A real-valued function $\rho: \Omega \rightarrow \mathbb{R}$ on a domain $\Omega$ is said to be an exhaustion function if, for every $\alpha \in \mathbb{R}$, the set $\rho^{-1}((-\infty, \alpha])$ is compact - that is, the sublevel sets of $\rho$ are compact.

Exhaustion functions of course always exist on domains and indeed on manifolds in general. One for (not necessarily bounded) domains that frequently occurs in complex analysis is $\max \left(\|z\|^{2},-\log \operatorname{dist}(z\right.$, the complement of the domain)). Exhaustion functions with special properties play an important role, for instance, in the study of Stein manifolds; these are of course more difficult to construct.

Now suppose that $G$ is a compact group of diffeomorphisms on a domain $\Omega$ and suppose that $\rho$ is an exhaustion function on $\Omega$. Then the function $\hat{\rho}$ defined by

$$
\hat{\rho}(z):=\int_{G} \rho(g(z)) d \lambda(g),
$$

where $d \lambda$ is the normalized Haar measure on $G$, is also an exhaustion function, as one easily sees. This function is $G$-invariant in the sense that $\hat{\rho}(g(z))=\hat{\rho}(z)$. Thus its sublevel sets are invariant under the action of $G$ : a given sublevel set is mapped to itself by each element of $G$.

If $\rho$ is $C^{\infty}$, then $\hat{\rho}$ is also $C^{\infty}$. In this case, for all sufficiently large $\alpha$, except for a set of measure 0 , the sublevel set $\hat{\rho}^{-1}(-\infty, \alpha]$ is a compact $C^{\infty}$ manifoldwith-boundary. This follows from Sard's theorem: one need only take $\alpha$ so large that the sublevel set is nonempty and such that $\alpha$ is a regular value for $\hat{\rho}$. 
Now we return to the situation of a sequence of compact groups $G_{j}$ converging in our previous sense to a compact group $G_{0}$. As in the general setting above, we choose a $C^{\infty}$ exhaustion function $\rho_{0}$ and average it over $G_{0}$ to get a $G_{0}$-invariant, $C^{\infty}$ exhaustion function $\hat{\rho}_{0}$.

Because $G_{j}$ is defined on $\Omega_{j}$ while $\hat{\rho}_{0}$ is defined on $\Omega_{0}$, we cannot average $\hat{\rho}_{0}$ to make it $G_{j}$-invariant. We can, however, perform the averaging on arbitrary compact subsets.

Specifically, choose $\alpha$ as above, so that $\hat{\rho}_{0}^{-1}(-\infty, \alpha]$ is nonempty and of course is a compact subset of $\Omega_{0}$. Let $L$ be a compact subset of $\Omega_{0}$ which contains $\hat{\rho}_{0}^{-1}(-\infty, \alpha]$ in its interior and let $L_{1}$ be a compact subset of $\Omega_{0}$ that contains $L$ in its interior.

Because the sequence $G_{j}$ converges to $G_{0}$, it follows easily that, for $j$ sufficiently large, the images under $G_{j}$ of points of $L$ lie in $L_{1}$. It then follows in addition that one can average the function $\rho_{0}$ over the action of $G_{j}$, as in the process of averaging to construct $\hat{\rho}$. Denote this new function on $L$ by $\hat{\rho}_{j}$. Note that, because the elements of $G_{j}$ are, for $j$ large, close to those of $G_{0}$, the function $\hat{\rho}_{j}$ is $C^{\infty}$-close (i.e., $\gamma_{L}$-close) to $\hat{\rho}_{0}$ on $L$. In particular, the sublevel set $L_{1} \cap \hat{\rho}_{j}^{-1}(-\infty, \alpha]$ will be, for $j$ sufficiently large, a smooth manifold-with-boundary which is $C^{\infty}$ close to $\hat{\rho}_{0}^{-1}(-\infty, \alpha]$.

In particular, if we choose a regular value $\alpha$ for $\hat{\rho}_{0}$ with the sublevel set $M_{0}:=$ $\hat{\rho}_{0}^{-1}(-\infty, \alpha]$ nonempty then, for all $j$ sufficiently large, the sublevel set $M_{j}:=$ $\hat{\rho}_{j}^{-1}(-\infty, \alpha]$ will be a nonempty $C^{\infty}$ manifold-with-boundary. Moreover it will be close to $\hat{\rho}_{0}^{-1}(-\infty, \alpha]$ in the $C^{\infty}$ sense. Namely, there will be a sequence of diffeomorphisms $\phi_{j}: M_{0} \rightarrow M_{j}$ which converges in the $C^{\infty}$ sense to the identity on $M_{0}$.

More precisely, these diffeomorphisms can be obtained as follows: for $j$ large, $\hat{\rho}_{j}$ has derivative bounded away from 0 along integral curves of the gradient of $\hat{\rho}$ (gradient relative to an arbitrary Riemannian metric, indeed) near $\hat{\rho}^{-1}(\{\alpha\})$. Motion along the integral curves gives a diffeomorphism of the $\alpha$-level of $\hat{\rho}$ onto the $\alpha$-level of $\hat{\rho}_{j}$. Standard Morse theory then establishes a diffeomorphism of $\hat{\rho}^{-1}([\beta, \alpha])$ onto $\hat{\rho}_{j}^{-1}([\beta, \alpha])$ for some $\beta<\alpha$ but with $\beta$ close to $\alpha$. This diffeomorphism is $C^{\infty}$ close to the identity and can hence be patched via a partition of unity to the identity diffeomorphism on $\tilde{\rho}^{-1}\left(\left[\frac{1}{2}(\alpha+\beta), \alpha\right]\right)$ to give the desired diffeomorphism of $\tilde{\rho}^{-1}((-\infty, \alpha])$ to $\tilde{\rho}_{j}^{-1}((-\infty, \alpha]), C^{\infty}$ close to the identity.

The next step of the proof is to form the doubles of the invariant subdomains with smooth boundary and extend the compact group actions to them. This will make it possible to apply the lemma above to the present situation.

For this, suppose that $\Omega$ is a domain, $M$ a compact subset that is a (nonempty) smooth manifold with boundary and $H$ a compact group of diffeomorphisms of $\Omega$ that map $M$ to itself. By the usual averaging process, similar to the construction of the invariant exhaustion functions as already discussed, there is a Riemannian metric 
$g$ on $\Omega$ for which the elements of $H$ act as isometries; i.e., $H$ is contained in $\operatorname{Isom}(g)$. Now the metric $g$ restricted to $M$ can be modified so as to remain invariant under $H$ while being a product metric at and near the boundary of $M$ (see [Greene and Krantz 1982a] for an early instance of this construction). This modification is obtained by first noting that, if $N$ is the inward unit normal (relative to $g$ ) along the boundary $\partial M$, then there is an $\epsilon>0$ such that the $g$-exponential map $E: \partial M \times[0, \epsilon) \rightarrow M$ defined by $E(p, s)=\exp _{p}(s N(p))$ is a diffeomorphism for $|s|<\epsilon$ and moreover $E(p, s), p \in \partial M, 0 \leq s<\epsilon$, is a diffeomorphism of manifolds with boundary onto a neighborhood $V$ of $\partial M$ in $M$. This is the usual tubular neighborhood construction.

Then one obtains a product metric $h$ on the neighborhood of the boundary as

$$
h=d s^{2}+d p^{2}
$$

where $d p^{2}$ is the metric on $\partial M$ and we push this metric over via $E$ to the neighborhood $V$ of $\partial M$ in $M$. This is clearly invariant under $H$. Then one can extend this metric to all of $M$ in an $H$-invariant way, by taking a function $\phi$ on $V$ that depends on $s$ alone and hence is invariant under the $H$-action. This function is to be 1 in a neighborhood of $s=0$, and hence as a function on $M$, is equal to 1 in a neighborhood of $\partial M$. It is to be equal to 0 when $s>\epsilon / 2$. Then $\phi h+(1-\phi) g$ will be a metric on $M$ as desired: it is smooth on all of $M$, is invariant under $H$, and is a product metric near $\partial M$.

This metric now extends smoothly to be a metric $\hat{h}$ on the double $\hat{M}$ of $M$ in an obvious way. The group $H$ acts on $\widehat{M}$ as a subgroup of the isometry group of $\hat{h}$. This subgroup of the isometry group of $\hat{h}$ will be denoted by $\hat{H}$.

Our construction can clearly be taken to be stable with respect to the original $H$-invariant metric $g$ on $M$ in the sense that, if $g_{1}$ is another $H$-invariant metric on $M$ which is $C^{\infty}$ close to $g$, then the corresponding metric $\hat{h}_{1}$ on the double $\widehat{M}$ of $M$ will be $C^{\infty}$ close to $\hat{h}$.

With these ideas in mind, we return to the convergence situation as before. Namely, we continue to denote by $\widehat{M}_{j}$ the doubles of the $G_{j}$-invariant sublevel sets, and let $\widehat{G}_{j}$ denote the extension of the $G_{j}$. Now, when $j$ is large, there are diffeomorphisms $\beta_{j}: \widehat{M}_{0} \rightarrow \widehat{M}_{j}$ which have the property that the pullback to $\widehat{M}_{0}$ of the $G_{j}$-action on $M_{j}$ via $\beta_{j}$ converges in the sense of Lemma 2.4 above.

In particular, $G_{j}$ is then isomorphic to a subgroup of $G_{0}$, for all sufficiently large $j$. Note that, as such, these isomorphisms apply not to $G_{j}$ itself but to the restriction of $G_{j}$ to $M_{j}$. But, since $M_{j}$ has nonempty interior, the restriction of $G_{j}$ to be an action on the $\left(G_{j}\right.$-invariant) set $M_{j}$ is injective: two isometries of a connected manifold which are equal on a nonempty open set are equal. (This follows easily by a standard continuation argument.) Hence the original $G_{j}$ are indeed isomorphic to a subgroup of $G_{0}$ when $j$ is sufficiently large. Thus the proposition is established. 


\section{Bergman metric and curvature with $C^{2}$ stability near the strongly pseudoconvex boundary}

Let $n>1$ throughout this section. Denote by $\mathscr{D}_{n}$ the collection of bounded domains in $\mathbb{C}^{n}$ with $C^{2}$ smooth, strongly pseudoconvex boundary, equipped with the $C^{2}$ topology via the $C^{2}$ topology on defining functions. The goal of this section is to establish the following result, which is Klembeck's theorem [1978] for domains in $\mathscr{D}_{n}$, with $C^{2}$ stability. In the statement below the notation $S_{\Omega}(p ; \xi)$ denotes the holomorphic sectional curvature of the Bergman metric of the domain $\Omega$ at $p$ along the holomorphic section generated by the tangent vector $\xi$.

Theorem 3.1. Let $\widehat{\Omega} \in \mathscr{D}_{n}$. Then, for every $\epsilon>0$, there exist $\delta>0$ and an open neighborhood $U$ of $\widehat{\Omega}$ in $\mathscr{D}_{n}$ such that, whenever $\Omega \in \mathcal{U}$,

$$
\sup \left\{\left|S_{\Omega}(p ; \xi)-\left(-\frac{4}{n+1}\right)\right|: \Omega \in U, \xi \in \mathbb{C}^{n} \backslash\{0\}\right\}<\epsilon
$$

for any $p \in \Omega$ satisfying $\operatorname{dis}\left(p, \mathbb{C}^{n} \backslash \Omega\right)<\delta$.

We remark, before giving the proof, that this result is crucial in establishing the semicontinuity theorem (Theorem 5.2): if $\widehat{\Omega}$ is not biholomorphic to the unit open ball, there exists $(\hat{p}, \hat{\xi}) \in T \Omega_{0}=\Omega_{0} \times \mathbb{C}^{n}$ such that $S_{\widehat{\Omega}}(\hat{p}, \hat{\xi}) \neq-4 /(n+1)$ due to Lu Qi-Keng's theorem [Lu 1966; Greene et al. 2011, Theorem 4.2.2]. Now Theorem 3.1 implies that, choosing $U$ smaller if necessary, this curvature difference continues to hold for every domain $\Omega \in \mathcal{U}$. Consequently, one sees that there exist a constant $\delta>0$ depending only on $(\hat{p}, \hat{\xi})$ and a neighborhood $U$ of $\widehat{\Omega}$ in the space of domains such that

$$
\operatorname{dis}\left(\phi(\hat{p}), \mathbb{C}^{n} \backslash \Omega\right)>\delta \quad \text { for all } \phi \in \operatorname{Aut}(\Omega)
$$

for every $\Omega \in \mathcal{U}$, a crucial point in the proof of Theorem 5.2.

Proof. It suffices to show that the following cannot hold:

$(\dagger)$ there exist $\epsilon_{0}>0$ and $\left\{\Omega_{v}\right\} \subset \mathscr{D}_{n}$ such that $\Omega_{v} \rightarrow \widehat{\Omega}$ in the $C^{2}$ topology as $v \rightarrow \infty$ and there exists a sequence $\left\{p_{v} \in \Omega_{v}\right\}$ with $\lim _{v \rightarrow \infty} \operatorname{dis}\left(p_{v}, \partial \Omega_{v}\right)=0$ such that

$$
\left|S_{\Omega_{v}}\left(p_{v}, \xi_{v}\right)+\frac{4}{n+1}\right| \geq \epsilon_{0}
$$

for every $v$.

Since the goal is to show that

$$
\lim _{\nu \rightarrow \infty}\left|S_{\Omega_{v}}\left(p_{v}, \xi_{v}\right)+\frac{4}{n+1}\right|=0,
$$

we may assume without loss of generality that $\lim _{v \rightarrow \infty} p_{v}$ exists. Denote this limit by $\hat{p}$. Notice that $\hat{p} \in \partial \widehat{\Omega}$. 
Let $q_{v} \in \partial \Omega_{v}$ be the closest boundary point of $\Omega_{v}$ to $p_{v}$ for every $v=1,2, \ldots$ Then consider a sequence $R_{v}: \mathbb{C}^{n} \rightarrow \mathbb{C}^{n}$ of complex rigid motions (i.e., unitary maps followed by translations) in $\mathbb{C}^{n}$ and another rigid motion $\widehat{R}$ satisfying

(1) $\hat{R}(\hat{p})=0$ and $R_{v}\left(q_{v}\right)=0$ for every $v$;

(2) $R_{v}\left(\partial \Omega_{v}\right)$ for every $v$, and $\hat{R}(\partial \hat{\Omega})$ are tangent at 0 to the hyperplane defined by $\operatorname{Re} z_{1}=0$;

(3) $\lim _{v \rightarrow \infty}\left\|R_{v}-\widehat{R}\right\|_{C^{2}}=0$, where the norm here is the $C^{2}$-norm of mappings on an open neighborhood of the closure of $\widehat{\Omega}$ in $\mathbb{C}^{n}$.

Notice that $R_{v}\left(\Omega_{v}\right)$ converges to $\hat{R}(\widehat{\Omega})$ in the $C^{2}$ topology on bounded domains with smooth boundaries. Therefore, without loss of generality, we may also assume the following:

$\left(1^{\prime}\right) 0 \in \partial \hat{\Omega} \cap\left(\bigcap_{\nu=1}^{\infty} \partial \Omega_{\nu}\right)$;

(2') $\partial \widehat{\Omega}$ and $\partial \Omega_{v}$ (for every $v=1,2, \ldots$ ) share the same outward normal vector $\mathbf{n}=(-1,0, \ldots, 0)$ at the origin;

$\left(3^{\prime}\right) p_{v}=\left(r_{v}, 0, \ldots, 0\right)$ with $r_{v}>0$ for every $v$.

Now we need the following three lemmas for the proof. The first is:

Lemma 3.2 ([Kim and Yu 1996]; cf. [Greene et al. 2011, Chapter 10]). There exists an open neighborhood $U$ of the origin in $\mathbb{C}^{n}$ such that

$$
\lim _{\nu \rightarrow \infty} \sup _{0 \neq \xi \in \mathbb{C}^{n}}\left|\frac{2-S_{\Omega_{\nu} \cap U}\left(p_{\nu} ; \xi\right)}{2-S_{\Omega_{v}}\left(p_{\nu} ; \xi\right)}-1\right|=0 .
$$

The proof of this lemma is a normal families argument.

Notice that this lemma implies: if $\lim _{v \rightarrow \infty} S_{\Omega_{v} \cap U}\left(p_{v} ; \xi\right)$ exists, it will coincide with $\lim _{\nu \rightarrow \infty} S_{\Omega_{v}}\left(p_{\nu} ; \xi\right)$.

The next two lemmas convert the problem of understanding the boundary asymptotic behavior of the Bergman curvature to that of the stability of the Bergman kernel function in the interior under perturbation of the boundary:

Lemma 3.3 ([Kim and Yu 1996]; cf. [Greene et al. 2011, Chapter 10]). Let the sequence $\left\{\left(p_{v} ; \xi_{v}\right) \in \Omega_{v} \times\left(\mathbb{C}^{n} \backslash\{0\}\right)\right\}$ be chosen as above. Let $B^{n}$ denote the open unit ball in $\mathbb{C}^{n}$. Then there exists a sequence of injective holomorphic mappings $\sigma_{v}: \Omega_{v} \cap U \rightarrow \mathbb{C}^{n}$ with the following properties:

(i) $\sigma_{v}\left(p_{v}\right)=0\left(\right.$ the origin of $\left.\mathbb{C}^{n}\right)$;

(ii) for every $r$ with $0<r<1$, there exists $N>0$ such that, for every $v>N$,

$$
(1-r) B^{n} \subset \sigma_{\nu}\left(\Omega_{v} \cap U\right) \subset(1+r) B^{n} .
$$

The third and last lemma toward the proof of Theorem 3.1 is as follows: 
Lemma 3.4 ([Ramadanov 1967; Kim and Yu 1996]; cf. [Greene et al. 2011, Chapter 10]). Let $D$ be a bounded domain in $\mathbb{C}^{n}$ containing the origin 0 . Let $\left\{D_{v}\right\}$ denote a sequence of bounded domains in $\mathbb{C}^{n}$ that satisfies the following convergence condition:

given $\epsilon>0$, there exists $N>0$ such that $(1-\epsilon) D \subset D_{\nu} \subset(1+\epsilon) D$ for every $v>N$.

Then, for every compact subset $F$ of $D$, the sequence of Bergman kernel functions $K_{D_{v}}$ of $D_{v}$ converges uniformly to the Bergman kernel function $K_{D}$ of $D$ on $F \times F$.

This is a result of Ramadanov [1967]. Now we return to the proof of Theorem 3.1.

Let $q_{v}, \xi_{v}, \hat{\Omega}, \Omega_{v}$ be as above. Let $U$ be an open neighborhood of the origin as in Lemma 3.2. Taking a subsequence, we may assume that $q_{v} \in \Omega_{v} \cap U$ for every $v$. Select $\sigma_{v}$ as in Lemma 3.3.

Apply Lemma 3.4 to our setting, with $D_{v}=\sigma_{v}\left(\Omega_{v} \cap U\right)$ and $D=B^{n}$. The conclusion of Lemma 3.4 states that the sequence $K_{D_{v}}(z, \zeta)$ converges uniformly to $K_{D}(z, \zeta)$ on $F \times F$. This of course implies that the sequence $K_{D_{v}}(z, \bar{\zeta})$ converges to $K_{D}(z, \bar{\zeta})$. Notice that the functions now involved are holomorphic functions in the $z$ and $\zeta$ variables together. Therefore Cauchy estimates imply that $K_{D_{v}}(z, \zeta)$ converges uniformly to $K_{D}(z, \zeta)$ on $F \times F$ in the $C^{k}$ sense for any positive integer $k$. Since the holomorphic sectional curvature of the Bergman metric involves derivatives of the Bergman kernel function up to fourth order, we may conclude that $S_{\sigma_{v}\left(\Omega_{v} \cap U\right)}(0 ; \cdot)$ converges uniformly to $S_{B^{n}}(0 ; \cdot)$ on $\left\{\xi \in \mathbb{C}^{n}:\|\xi\|=1\right\}$. Notice that the latter is the constant function with value $-4 /(n+1)$.

Combining this result with the localization lemma (Lemma 3.2), the conversion lemma (Lemma 3.3), and the fact that every biholomorphism is an isometry for the Bergman metric, we see that

$$
\begin{aligned}
-\frac{4}{n+1} & =\lim _{\nu \rightarrow \infty} S_{\sigma_{\nu}\left(\Omega_{v} \cap U\right)}\left(0 ;\left.d \sigma_{\nu}\right|_{q_{\nu}}\left(\xi_{v}\right)\right)=\lim _{\nu \rightarrow \infty} S_{\sigma_{\nu}\left(\Omega_{v} \cap U\right)}\left(\sigma_{\nu}\left(q_{v}\right) ;\left.d \sigma_{\nu}\right|_{q_{\nu}}\left(\xi_{v}\right)\right) \\
& =\lim _{\nu \rightarrow \infty} S_{\Omega_{\nu} \cap U}\left(q_{\nu} ; \xi_{\nu}\right)=\lim _{\nu \rightarrow \infty} S_{\Omega_{\nu}}\left(q_{\nu} ; \xi_{v}\right) .
\end{aligned}
$$

This completes the proof of Theorem 3.1.

Remark 3.5 (completeness of the Bergman metric). The Bergman metric of a bounded strongly pseudoconvex domain is known to be complete ([Diederich 1973]; for the more general case see [Ohsawa 1981]). Since the scaled limit shown in the proof of Lemma 3.3 is the unit ball, a variation of that proof argument also yields the same conclusion as [Diederich 1973] regarding completeness also (see [Greene et al. 2011, Section 10.1.7]). 


\section{Stable $\boldsymbol{C}^{\boldsymbol{k}}$-extension of automorphisms}

The purpose of this section is to establish the stability of the extension theorem for the automorphisms of a bounded strongly pseudoconvex domain under $C^{k}$ perturbation for finite $k$.

The result and the techniques involved in the proofs are new. More importantly, the contents of this section (especially Theorem 4.3 on page Theorem 4.3) are essential in creating the necessary "metric double" in the proof of Theorem 5.2.

Convergence of Lempert's representative map. Let $X, Y$ be complex Banach spaces. Let $\phi: U \rightarrow Y$ be a map from an open subset $U$ of $X$ into $Y$. The map $\phi$ is said to be differentiable at $x \in X$, if there exists a bounded linear map $D_{x} \phi: X \rightarrow Y$ such that

$$
\left\|\phi(x+h)-\phi(x)-\left(D_{x} \phi\right)(h)\right\|_{Y}=o\left(\|h\|_{X}\right)
$$

as $\|h\|_{X} \rightarrow 0$. Let $L(X, Y)$ denote the set of bounded linear maps from $X$ into $Y$. It is naturally equipped with the operator norm and hence becomes a Banach space. Then $\phi$ is said to be $C^{1}$ on $U$ if $D_{x} \phi$ exists for all $x \in U$ and

$$
D \phi: x \in U \mapsto D_{x} \phi \in L(X, Y)
$$

is continuous.

It is also well established what it means for $\phi$ to belong to the class $C^{k}$; see, [Mujica 1986], for example. To understand this point, consider the space $L(X \times \cdots \times$ $X, Y)$ of bounded $k$-linear maps with values in $Y$. For an $S \in L(X \times \cdots \times X, Y)$, define its norm as follows:

$$
\|S\|_{k}=\sup \left\{\left\|S\left(h_{1}, \ldots, h_{k}\right)\right\|_{Y}:\left\|h_{1}\right\|_{X} \leq 1, \ldots,\left\|h_{k}\right\|_{X} \leq 1\right\} .
$$

One more piece of notation is necessary: for a $k$-linear map $S$, a $(k-1)$-linear map $[S](h)$ is defined by

$$
[S](h)\left(h_{1}, \ldots, h_{k-1}\right):=S\left(h, h_{1}, \ldots, h_{k-1}\right) .
$$

Now the idea of a map belonging to the class $C^{k}$ can be defined inductively: the map $\phi$ is said to be $C^{k}$ at $x \in X$, for $k=1,2, \ldots$, if there exits a bounded $k$-linear map $D_{x}^{k} \phi: \underbrace{X \times \cdots \times X}_{k} \rightarrow Y$ such that

$$
\left\|D_{x+h}^{k-1} \phi-D_{x}^{k-1} \phi-\left[D_{x}^{k} \phi\right](h)\right\|_{k-1}=o\left(\|h\|_{X}\right)
$$

as $h \rightarrow 0$ and

$$
D^{k} \phi: x \in U \mapsto D_{x}^{k} \phi \in L(\underbrace{X \times \cdots \times X}_{k}, Y)
$$


is continuous. It is also known that such a $D_{x}^{k} \phi$ is symmetric $k$-linear.

Similarly, we may define the concept of Hölder class. For an $\alpha$ with $0<\alpha \leq 1$, a map $\phi$ is said to belong to the class $C^{k, \alpha}$ if $\phi$ is $C^{k}$ and

$$
\sup _{\substack{x, y \in U \\ x \neq y}} \frac{\left\|D_{x}^{k} \phi-D_{y}^{k} \phi\right\|_{k}}{\|x-y\|_{X}^{\alpha}}<\infty .
$$

Throughout this section, we denote by $\Delta$ the open unit disc $\{z \in \mathbb{C}:|z|<1\}$. We shall follow the terminology of [Lempert 1986] closely. Let $s$ be such that $0<s<\alpha$ and set

$X_{n}=\left\{f: \partial \Delta \rightarrow \mathbb{C}^{n} \mid f \in C^{0, s}\right\}$,

$Y_{n}=\left\{f \in X_{n}: f\right.$ admits a holomorphic continuation to $\left.\operatorname{cl}(\Delta)\right\}$,

$Y_{n}^{\perp}=\left\{f \in X_{n}: f\right.$ admits an antiholomorphic continuation to $\operatorname{cl}(\Delta)$ with $\left.f(0)=0\right\}$.

Notice that $X_{n}=Y_{n} \oplus Y_{n}^{\perp}$.

Let $\Omega=\Omega_{\rho}$ be a bounded strictly convex domain defined by the $C^{k+1, \alpha}$ defining function $\rho$. Then there exists a convex open neighborhood $V$ of $\operatorname{cl}(\Omega)$ such that $\Omega=\Omega_{\rho}=\{z \in V: \rho(z)<0\}$, where the defining function $\rho: U \rightarrow \mathbb{R}$, defined on a convex open set $U$ with $\operatorname{cl}(V) \subset U$, is of class $C^{k+1, \alpha}(k \geq 1,0<\alpha<1)$ with $d \rho \neq 0$ at any point of $\partial \Omega$. We may further assume without loss of generality that

(1) $\rho: U \rightarrow \mathbb{R}$ is compactly supported, and

(2) the real Hessian of $\rho$ is strictly positive at every point of $\partial \Omega$.

Let $\mathcal{N}$ be a $C^{k+1, \alpha}$ neighborhood of $\rho$ chosen so small that every element of $\mathcal{N}$ has its real Hessian strictly positive at every point of $V$. We may require further that there exists a constant $R^{\prime}>0$ such that, if $\eta, \tau \in \mathcal{N}$, then $\|\eta-\tau\|_{C^{k+1, \alpha}(U)}<1$ and $\|\eta\|_{C^{k+1, \alpha}(U)}<R^{\prime}$.

Let $p$ be a point in $\Omega$ and let $W$ a neighborhood of $p$ in $\Omega$ such that $W \subset \Omega_{\eta}$ for all $\eta \in \mathcal{N}$. Define $\Theta: \mathcal{N} \oplus\left(\mathbb{C}^{n} \backslash\{0\}\right) \oplus W \rightarrow Y_{n}$ by $\Theta(\eta, \zeta, q)=e_{\eta, \zeta, q}$, where $e_{\eta, \zeta, q}$ is the stationary map (i.e., extremal map) from $\operatorname{cl}(\Delta)$ to $\operatorname{cl}\left(\Omega_{\eta}\right)$ satisfying $e_{\eta, \zeta, q}(0)=q$ and $e_{\eta, \zeta, q^{\prime}}(0)=\mu \zeta$ for some $\mu>0$.

Proposition 4.1. The map $\Theta$ is locally $C^{k, \alpha-s}$ for any $0<s<\alpha$.

Proof. Let $(\eta, v, q) \in \mathcal{N} \oplus \mathbb{C}^{n} \backslash\{0\} \oplus \mathscr{W}$. We shall prove that $\Theta$ is $C^{k, \alpha-s}$ near $(\eta, v, q)$. Let $e=e_{\eta, v, q}=\left(e_{1}, \ldots, e_{n}\right): \operatorname{cl}(\Delta) \rightarrow \operatorname{cl}\left(\Omega_{\eta}\right)$ and $\tilde{e}=\left(\tilde{e}_{1}, \ldots, \tilde{e}_{n}\right)$ be the dual map of $e$. (See [Lempert 1981] for the definition of the dual map and its basic properties.) Since $\tilde{e}$ has no zeros, there exist two components which do not vanish simultaneously by a generic linear change of coordinates. Hence we may assume without loss of generality that $\tilde{e}_{1}$ and $\tilde{e}_{2}$ do not vanish simultaneously on $\operatorname{cl}(\Delta)$. It is also shown in [Lempert 1981] that $\tilde{e}$ extends to a $C^{k, \alpha}$ map up to the 
boundary, and that there exist functions $G_{1}, G_{2} \in C^{k, \alpha}(\operatorname{cl}(\Delta))$ that are holomorphic in $\Delta$ and satisfy $\tilde{e}_{1} G_{1}+\tilde{e}_{2} G_{2} \equiv 1$. Define the holomorphic matrix $H$ on $\Delta$ by

$$
H=\left(\begin{array}{ccccc}
e_{1}^{\prime} & -\tilde{e}_{2} & -G_{1} \tilde{e}_{3} & \cdots & -G_{1} \tilde{e}_{n} \\
e_{2}^{\prime} & \tilde{e}_{2} & -G_{2} \tilde{e}_{3} & \cdots & -G_{2} \tilde{e}_{n} \\
e_{3}^{\prime} & 0 & 1 & \cdots & 0 \\
\vdots & \vdots & \vdots & \ddots & \vdots \\
e_{n}^{\prime} & 0 & 0 & \cdots & 1
\end{array}\right) .
$$

Notice that $H \in C^{0, s}(\operatorname{cl}(\Delta))$ and $\operatorname{det}(H) \neq 0$ on $\operatorname{cl}(\Delta)$. Set

$$
Y_{n}^{R, U}=\left\{f \in Y_{n}:\|f\|_{C^{1}(\mathrm{cl}(\Delta))}<R, f(\partial \Delta) \subset U\right\}
$$

and define the map

$$
\Phi: \mathcal{N} \oplus \mathbb{C}^{n} \backslash\{0\} \oplus \mathcal{W} \oplus Y_{n}^{R, U} \oplus \mathbb{R} \rightarrow T \oplus Y_{n-1}^{\perp} \oplus \mathbb{C}^{n} \oplus \mathbb{C}^{n}
$$

by

$$
\Phi(r, v, q, f, \lambda)=\left(r \circ f, \pi\left(\frac{\left\langle H^{t} r_{z} \circ f\right\rangle}{\left(H^{t} r_{z} \circ f\right)_{1}}\right), f(0)-q, f^{\prime}(0)-\lambda v\right),
$$

where

(i) $T=\left\{g: \partial \Delta \rightarrow \mathbb{R}: g \in C^{0, s}\right\}$,

(ii) $\pi: Y_{n-1} \rightarrow Y_{n-1}^{\perp}$ is defined by $\pi\left(\sum_{-\infty}^{\infty} a_{k} z^{k}\right)=\sum_{-\infty}^{-1} a_{k} z^{k}$, and

(iii) $\left(H^{t} r_{z} \circ f\right)_{j}$ denotes the $j$-th component of $H^{t} r_{z} \circ f$ and $\left\langle H^{t} r_{z} \circ f\right\rangle=$ $\left(\left(H^{t} r_{z} \circ f\right)_{2}, \ldots,\left(H^{t} r_{z} \circ f\right)_{n}\right)$.

Then $f: \operatorname{cl}(\Delta) \rightarrow \operatorname{cl}\left(\Omega_{r}\right)$ is an extremal map satisfying $f(0)=q, f^{\prime}(0)=\lambda v$ if and only if $\Phi(r, v, q, f, \lambda)=0$. So, according to [Lempert 1986], we only need to prove that $\Phi$ is $C^{k, \alpha-s}$. For this purpose define the map $\Psi: \mathcal{N} \oplus Y_{n}^{R, U} \rightarrow T$ by $\Psi(r, f)=r \circ f$. Then we pose the following:

Claim. $\Psi$ is $C^{k, \alpha-s}$.

We shall prove this claim by induction on $k$. We need some notation. For a domain $\Omega, k \in \mathbb{Z}^{+}$, and $0<\alpha \leq 1$, define

$$
\|g\|_{C^{k, \alpha}(\operatorname{cl}(\Omega))}=\sup _{\substack{x \in \mathrm{cl}(\Omega) \\|\gamma|=0,1, \ldots, k}}\left|D^{\gamma} g(x)\right|+\sup _{\substack{x, y \in \mathrm{cl}(\Omega) \\ x \neq y,|\gamma|=k}} \frac{\left|D^{\gamma} g(x)-D^{\gamma} g(y)\right|}{|x-y|^{\alpha}} .
$$

Moreover, $A \lesssim B$ will mean that $A \leq C B$ for some constant $C$. In turn, $A \lesssim B$ will mean that $A \rightarrow 0$ whenever $B \rightarrow 0$.

Let $j \in\{0, \ldots, k\}$. Let $\mathcal{N}_{j}=\left\{r \in C^{j+1, \alpha}(U):\|r\|_{C^{j+1, \alpha}(U)}<R^{\prime}\right\}$. Define $\Psi_{j}: \mathcal{N}_{j} \oplus Y_{n}^{R, U} \rightarrow T$ by $\Psi_{j}(r, f)=r \circ f$. Suppose that, for all $r, \tau \in \mathcal{N}_{j}$, we have $\|r-\tau\|_{C^{j, \alpha}(U)}<1$. 
In case $j=0$, it suffices to show that

$$
\left\|\Psi_{0}(r, f)-\Psi_{0}(\tau, g)\right\|_{C^{0, s}(\partial \Delta)} \lesssim\left(\|r-\tau\|_{C^{0, \alpha}(U)}+\|f-g\|_{C^{0, s}(\partial \Delta)}\right)^{\alpha-s} .
$$

For $x \in \partial \Delta$,

$$
\begin{aligned}
|r \circ f(x)-\tau \circ g(x)| & \leq|r \circ f(x)-r \circ g(x)|+|r \circ g(x)-\tau \circ g(x)| \\
& \lesssim|f(x)-g(x)|^{\alpha-s}+|(r-\tau) \circ g(x)| \\
& \lesssim\left(\|f-g\|_{C^{0, s}(\partial \Delta)}+\|r-\tau\|_{C^{0, \alpha}(U)}\right)^{\alpha-s} .
\end{aligned}
$$

For $x, y \in \partial \Delta$, let $\delta(x, y)=r \circ f(x)-\tau \circ g(x)-r \circ f(y)+\tau \circ g(y)$. Then

$$
\begin{aligned}
|\delta(x, y)| \leq & |r \circ f(x)-r \circ g(x)|+|r \circ g(x)-\tau \circ g(x)| \\
& \quad+|r \circ f(y)-r \circ g(y)|+|r \circ g(y)-\tau \circ g(y)| \\
\leq & 2\left(R^{\prime}\right)^{\alpha}|f(x)-g(x)|^{\alpha}+2\|r-\tau\|_{C^{0, \alpha}(U)} \\
\leq & 2\left(R R^{\prime}\right)^{\alpha}\|f-g\|_{C^{0, s}(\partial \Delta)}^{\alpha}+2\|r-\tau\|_{C^{0, \alpha}(U)}
\end{aligned}
$$

and

$$
\begin{aligned}
|\delta(x, y)| & \leq|r \circ f(x)-r \circ f(y)|+|\tau \circ g(x)-\tau \circ g(y)| \\
& \leq R^{\prime}|f(x)-f(y)|^{\alpha}+R^{\prime}|g(x)-g(y)|^{\alpha} \leq 2 R R^{\prime}|x-y|^{\alpha} .
\end{aligned}
$$

This implies that

$$
|\delta(x, y)| \lesssim\left(\|f-g\|_{C^{0, s}(\partial \Delta)}+\|r-\tau\|_{C^{0, \alpha}(U)}\right)^{\alpha-s}|x-y|^{s},
$$

which proves the case $j=0$.

Let $j>0$. Suppose that $\Psi_{j}: \mathcal{N}_{j} \oplus Y_{n}^{R, U} \rightarrow T$ is of class $C^{j, \alpha-s}(U)$. Then, since

$$
D_{(r, f)} \Psi_{j+1}(\tau, g)=\left(r^{\prime} \circ f\right) g+\tau \circ f=\Psi_{j}\left(r^{\prime}, f\right) g+\Psi_{j}(\tau, f),
$$

it follows that $\Psi_{j+1}$ is of $C^{j+1, \alpha-s}(U)$. This proves the claim.

Since $\pi$ is a bounded linear map, the second component of $\Phi$ is also of class $C^{k, \alpha-s}(U)$. The proof of the proposition is now complete.

Next, for $r \in \mathcal{N}, q \in W$, consider Lempert's representation map at $q$ for the domain $\Omega_{r}$. We have $L_{r, q}: \operatorname{cl}\left(\mathbb{B}^{n}\right) \rightarrow \operatorname{cl}\left(\Omega_{r}\right)$ defined by $L_{r, q}(\zeta)=\Theta(r, \zeta, q)(|\zeta|)=$ $e_{r, \zeta, q}(|\zeta|)$. The following proposition discusses the convergence of these representation maps.

Proposition 4.2. Let $\rho_{j}, \rho \in \mathcal{N}$ and $p_{j}, p \in W$ be such that $\left\|\rho_{j}-\rho\right\|_{C^{k+1, \alpha}(U)} \rightarrow 0$, $\left|p_{j}-p\right| \rightarrow 0$ as $j \rightarrow \infty$. Set the notation $L_{j}:=L_{\rho_{j}, p_{j}}, L:=L_{\rho, p}$ and $\mathbb{B}_{\delta}^{n}:=$ $\mathbb{B}^{n} \backslash\left\{z \in \mathbb{C}^{n}:|z|<\delta\right\}$. Then, for $0<\beta<\alpha$ and $0<\delta<1$, Lempert's representation 
maps $L_{j}$ for $\Omega_{\rho_{j}}$ converge to Lempert's representation map $L$ for $\Omega_{\rho}$ on $\mathbb{B}_{\delta}^{n}$ in the $C^{k, \beta}$ norm, as $j \rightarrow \infty$.

Proof. Let ev: $Y_{n} \rightarrow \mathbb{C}^{n}$ be defined by ev $(g)=g(1)$ (here "ev" stands for "evaluation" map). Since $L(\zeta)=\Theta(\rho, \zeta, p)(1)=\operatorname{ev} \circ \Theta(\rho, \zeta, p)$ for $\zeta \in \partial \mathbb{B}^{n}$, ev is bounded linear. Write $D^{\ell}=\partial^{m_{1}+\cdots+m_{n}} / \partial x_{1}^{m_{1}} \cdots \partial x_{n}^{m_{n}}$, where $|\ell|=m_{1}+\cdots+m_{n}$. Then

$$
D^{\ell} L(\zeta)=\left(D_{(\rho, \zeta, p)}^{|\ell|} \Theta\right)(\underbrace{\vec{x}_{1}, \ldots, \vec{x}_{1}}_{m_{1}} ; \ldots ; \underbrace{\vec{x}_{n}, \ldots, \vec{x}_{n}}_{m_{n}})(1) .
$$

So $\left\|L_{j}-L\right\|_{C^{k, \beta}\left(\partial \mathbb{B}^{n}\right)} \rightarrow 0$ as $j \rightarrow \infty$.

Given $v \in \mathbb{C}^{n},|v|=1, \xi \in \Delta$, denote by $e$ the extremal map satisfying $e(0)=p$, $e^{\prime}(0)=\mu v$ for some $\mu>0$. Then $L(\xi v)=e(\xi|v|)=e(\xi)$. This implies that $L(\xi v)$ is holomorphic with respect to $\xi$. Now the Poisson integral formula for $\Delta$ yields the desired conclusion.

A simultaneous extension theorem for automorphisms. The next goal is to establish the following theorem, which treats the $C^{k, \beta}$ convergence of sequences of automorphisms. This result is new, and the proof technique is new. It has independent interest.

Theorem 4.3 (uniform extension). Let $\Omega_{j}, \Omega$ be bounded, strongly pseudoconvex domains in $\mathbb{C}^{n}$ with $C^{k+1, \alpha}(k \in \mathbb{Z}, k \geq 2,0<\alpha \leq 1)$ boundaries such that $\Omega_{j}$ converges to $\Omega$ as $j \rightarrow \infty$ in the $C^{k+1, \alpha}$ topology, and with $\Omega$ not biholomorphic to the ball. Let a sequence $\left\{f_{j} \in \operatorname{Aut}\left(\Omega_{j}\right): j=1,2, \ldots\right\}$ be given. Then, for any $\beta$ with $0<\beta<\alpha$, the sequence $f_{j}$ (every one of which extends to a $C^{k, \beta}$ diffeomorphism of the closure $\mathrm{cl}\left(\Omega_{j}\right)$ by the "sharp extension theorem" of [Lempert 1986]) admits a subsequence $\Omega_{j_{\ell}}$ and $f_{j_{\ell}} \in \operatorname{Aut}\left(\Omega_{f_{\ell}}\right)$ that converges to the $C^{k, \beta}$-diffeomorphism, the extension of $f \in \operatorname{Aut}(\Omega)$, in the $C^{k, \beta}$ topology.

This indeed is a normal family theorem together with Hölder convergence up to the boundary. Of course precise definitions and terminology are in order, which will be presented here as the exposition progresses.

Definition 4.4. Let $\Omega_{j}$ and $\Omega$ be bounded strongly pseudoconvex domains in $\mathbb{C}^{n}$ with $C^{k, \alpha}(k \in \mathbb{Z}, k \geq 2,0<\alpha \leq 1)$ boundaries. As $j \rightarrow \infty$, the sequence of domains $\Omega_{j}$ is said to converge to $\Omega$ in the $C^{k, \alpha}$ topology, if there exist an open neighborhood $U$ of $\operatorname{cl}(\Omega), C^{k, \alpha}$ diffeomorphisms $F_{j}: U \rightarrow U$, and a positive integer $N$ such that

- $\operatorname{cl}(\Omega) \Subset U$;

- $\operatorname{cl}\left(\Omega_{j}\right) \Subset U$ for all $j>N$;

- each $F_{j}$ maps $\operatorname{cl}(\Omega)$ onto $\operatorname{cl}\left(\Omega_{j}\right)$ as a $C^{k, \alpha}$ diffeomorphism; for every $j>N$;

- $\left\|F_{j}-\mathrm{id}\right\|_{C^{k, \alpha}(U)} \rightarrow 0$ and $\left\|F_{j}^{-1}-\mathrm{id}\right\|_{C^{k, \alpha}(U)} \rightarrow 0$, as $j \rightarrow \infty$. 
In a similar manner, we say that the sequence of maps $f_{j} \in C^{k, \alpha}\left(\Omega_{j}, \mathbb{C}^{m}\right)$ converges to $f \in C^{k, \alpha}\left(\Omega, \mathbb{C}^{m}\right)$ in the $C^{k, \alpha}$ sense, if

$$
\lim _{j \rightarrow \infty}\left\|f_{j} \circ F_{j}-f\right\|_{C^{k, \alpha}(\Omega)}=0 .
$$

We now present several technical lemmas.

Lemma 4.5. Let $\Omega_{j}$ be a domain in $\mathbb{R}^{n_{j}}$ for each $j=1,2$, 3. If

(i) $g, h: \Omega_{1} \rightarrow \Omega_{2}$ are $C^{k^{\prime}, \alpha^{\prime}}$ maps that are injective,

(ii) $f: \Omega_{2} \rightarrow \Omega_{3}$ is a $C^{k^{\prime \prime}, \alpha^{\prime \prime}}$ map, and

(iii) $(k, \alpha)$ is the pair of the positive integer $k$ and the real number $\alpha$ satisfying $k+\alpha=\min \left\{k^{\prime}+\alpha^{\prime}, k^{\prime \prime}+\alpha^{\prime \prime}\right\}$ and $0<\alpha \leq 1$,

then

(1) $f \circ g \in C^{k, \alpha}\left(\Omega_{1}, \Omega_{3}\right)$ and

(2) $\|f \circ g-f \circ h\|_{C^{k, \beta}\left(\Omega_{1}\right)} \lesssim\|g-h\|_{C^{k, \alpha}\left(\Omega_{1}\right)}$ for any $\beta$ with $0<\beta<\alpha$.

Proof. We present the verification of (1) only, as our arguments are mostly straightforward computations and the proof of (2) is similar. The chain rule implies that

$$
D^{\ell}(f \circ g)(x)=\sum\left(D^{m} f\right)(g(x))\left(D^{m_{1}} g(x)\right)^{m_{1}^{\prime}}\left(D^{m_{2}} g(x)\right)^{m_{2}^{\prime}} \ldots\left(D^{m_{n}} g(x)\right)^{m_{n}^{\prime}},
$$

where $\ell$ and $m$ are multiindices and $m_{j}$ nonnegative integers satisfying $|m| \leq|\ell|$ and $\sum m_{j}^{\prime} \leq|\ell|$. (We use the usual multiindex notation here; we omit detailed expressions as they are standard.) Note that

$$
\|f \circ g\|_{C^{k, \alpha}}=\sup _{\substack{x \in \Omega_{1} \\ 0 \leq|\gamma| \leq k}}\left|D^{\gamma}(f \circ g)(x)\right|+\sup _{\substack{x, y \in \Omega_{1} \\ x \neq y,|\gamma|=k}} \frac{\left|D^{\gamma}(f \circ g)(x)-D^{\gamma}(f \circ g)(y)\right|}{|x-y|^{\alpha}} .
$$

First, one sees immediately that

$$
\sup _{\substack{x \in \Omega_{1} \\|\gamma|=0,1, \ldots, k}}\left|D^{\gamma}(f \circ g)(x)\right| \lesssim\|f\|_{C^{k, \alpha}\left(\Omega_{2}\right)} \sum\|g\|_{C^{k, \alpha}\left(\Omega_{1}\right)}^{m_{1}^{\prime}+\cdots+m_{n}^{\prime}}<\infty .
$$

On the other hand,

$$
\begin{aligned}
& \left|D^{\gamma}(f \circ g)(x)-D^{\gamma}(f \circ g)(y)\right| \\
& =\mid \sum\left\{D^{m} f(g(x)) \cdot\left(D^{m_{1}} g(x)\right)^{m_{1}^{\prime}} \ldots \cdot\left(D^{m_{n}} g(x)\right)^{m_{n}^{\prime}}\right. \\
& \left.\quad-D^{m} f(g(y)) \cdot\left(D^{m_{1}} g(y)\right)^{m_{1}^{\prime}} \cdots\left(D^{m_{n}} g(y)\right)^{m_{n}^{\prime}}\right\} \mid
\end{aligned}
$$




$$
\begin{aligned}
& \leq \sum\left\{\left|\left(D^{m} f(g(x))-D^{m} f(g(y))\right) \cdot\left(D^{m_{1}} g(x)\right)^{m_{1}^{\prime}} \cdots \cdot\left(D^{m_{n}} g(x)\right)^{m_{n}^{\prime}}\right|\right. \\
& \left.\quad+\mid\left(D^{m} f(g(y))\right) \cdot\left(\left(D^{m_{1}} g(x)\right)^{m_{1}^{\prime}}-D^{m_{1}} g(y)\right)^{m_{1}^{\prime}}\right) \\
& \quad \cdot\left(D^{m_{2}} g(x)\right)^{m_{2}^{\prime}} \cdots \cdot\left(D^{m_{n}} g(x)\right)^{m_{n}^{\prime}} \mid \\
& \quad+\cdots \\
& \left.\left.\quad+\mid\left(D^{m} f(g(y))\right) \cdot\left(D^{m_{1}} g(y)\right)^{m_{1}^{\prime}}\right) \cdots\left(\left(D^{m_{n}} g(x)\right)^{m_{n}^{\prime}}-\left(D^{m_{1}} g(y)\right)^{m_{1}^{\prime}}\right) \mid\right\} \\
& \lesssim\|f\|_{C^{k, \alpha}\left(\Omega_{2}\right)}\left(1+\|g\|_{C^{0}\left(\Omega_{1}\right)}^{\alpha}\right) P\left(\|g\|_{C^{k, \alpha}\left(\Omega_{1}\right)}\right)|x-y|^{\alpha},
\end{aligned}
$$

where $P$ is an appropriate polynomial with $P(0, \ldots, 0)=0$. Hence (1) follows. We omit the proof of (2).

Lemma 4.6. Let $k \geq 1$. Assume that $\Omega_{1}, \Omega_{2}$ are bounded domains in $\mathbb{R}^{n}$ admitting $C^{k, \alpha}$ diffeomorphisms $f_{j}, f: \operatorname{cl}\left(\Omega_{1}\right) \rightarrow \operatorname{cl}\left(\Omega_{2}\right)$ satisfying $\left\|f_{j}-f\right\|_{C^{k, \alpha}\left(\mathrm{cl}\left(\Omega_{1}\right)\right)} \rightarrow 0$ as $j \rightarrow \infty$. If $\lim _{j \rightarrow \infty} \sup _{x \in \mathrm{cl}\left(\Omega_{2}\right)}\left|f_{j}^{-1}(x)-f^{-1}(x)\right|=0$, then

$$
\lim _{j \rightarrow \infty}\left\|f_{j}^{-1}-f^{-1}\right\|_{C^{k, \beta}\left(\mathrm{cl}\left(\Omega_{2}\right)\right)}=0
$$

for any $0<\beta<\alpha$.

Proof. The inverse function theorem implies that $\left.d f_{j}^{-1}\right|_{f_{j}(y)}=\left(\left.d f_{j}\right|_{y}\right)^{-1}$ and $\left.d f^{-1}\right|_{f(y)}=\left(\left.d f\right|_{y}\right)^{-1}$. Since $\operatorname{cl}\left(\Omega_{1}\right)$ and $\operatorname{cl}\left(\Omega_{2}\right)$ are compact, there exist a constant $C>0$ and a positive integer $N$ such that $\left|\operatorname{det}\left(\left.d f\right|_{y}\right)\right|>C$ and $\left|\operatorname{det}\left(\left.d f_{j}\right|_{y}\right)\right|>C$ for any point $y \in \Omega_{1}$ and any integer $j>N$. Lemma 4.5 and its proof argument now yield the desired conclusion.

Lemma 4.7. Let $k$ be an integer with $k \geq 2$ and $\alpha$ a real number satisfying $0<\alpha \leq 1$. If $\Omega$ is a bounded, strongly pseudoconvex domain in $\mathbb{C}^{n}$, not biholomorphic to the unit open ball, with $C^{k+1, \alpha}$ boundary then, for any $\beta$ with $0<\beta<\alpha$, there exist an open neighborhood $U$ of $\Omega$ and a constant $C$ such that $\|f\|_{C^{k, \beta}\left(\operatorname{cl}\left(\Omega^{\prime}\right)\right)}<C$ for any $\Omega^{\prime} \in \mathcal{U}$ and any $f \in \operatorname{Aut}\left(\Omega^{\prime}\right)$.

Proof. Assume the contrary. Then there exists a sequence of strongly pseudoconvex domains $\Omega_{j}$ with $C^{k+1, \alpha}$ boundary converging to $\Omega$ in the $C^{k+1, \alpha}$ topology and a sequence $f_{j} \in \operatorname{Aut}\left(\Omega_{j}\right)$ such that

$$
\lim _{j \rightarrow \infty}\left\|f_{j}\right\|_{C^{k, \beta}\left(\operatorname{cl}\left(\Omega_{j}\right)\right)}=\infty .
$$

Then either

(1) there exists a sequence $\left\{x_{j} \in \Omega_{j}: j=1,2, \ldots\right\}$ such that $\left|D^{\gamma} f_{j}\left(x_{j}\right)\right| \rightarrow \infty$ as $j \rightarrow \infty$ for some multiindex $\gamma$ satisfying $0 \leq|\gamma| \leq k$; or

(2) there exist $x_{j}, y_{j} \in \Omega_{j}$ such that $\left|D^{\gamma} f_{j}\left(x_{j}\right)-D^{\gamma} f_{j}\left(y_{j}\right)\right| /\left|x_{j}-y_{j}\right|^{\beta}$ goes to infinity with $j$ for some multiindex $\gamma$ with $|\gamma|=k$. 
Suppose that (1) holds. Then, since the sequence $f_{j}$ converges to $f$ in the $C^{\infty}(K)$ topology on every compact subset $K$ of $\Omega$, it must be the case that $\lim _{j \rightarrow \infty} x_{j}=$ $p \in \partial \Omega$ (taking a subsequence if necessary).

We shall arrive at the desired contradiction to (1) by means of the following three steps:

Step 1. Adjustments. Let $F_{j}$ denote the same diffeomorphism of $\operatorname{cl}(\Omega)$ onto $\operatorname{cl}\left(\Omega_{j}\right)$ as in Definition 4.4. Set $F_{j}(p)=p_{j}, f_{j}\left(p_{j}\right)=q_{j}, f(p)=q$. Take the invertible affine $\mathbb{C}$-linear transformations $T, T_{j}, t, t_{j}: \mathbb{C}^{n} \rightarrow \mathbb{C}^{n}$ such that

- $T_{j}\left(p_{j}\right)=T(p)=t_{j}\left(q_{j}\right)=t(q)=(0, \ldots, 0)$;

- the outward normal vectors to the boundaries of $T_{j}\left(\Omega_{j}\right), T(\Omega), t_{j}\left(\Omega_{j}\right)$ and $t(\Omega)$ at $(0, \ldots, 0)$ are equal to $(1,0, \ldots, 0)$; and

- $\lim _{j \rightarrow \infty} T_{j}=T$ and $\lim _{j \rightarrow \infty} t_{j}=t$.

Then $T_{j}\left(\Omega_{j}\right)$ converges to $T(\Omega)$ in the $C^{k+1, \alpha}$ topology, and also $t_{j}\left(\Omega_{j}\right)$ converges to $t(\Omega)$. Replacing therefore $f$ and $f_{j}$, respectively, by $t \circ f \circ T^{-1}$ and $t_{j} \circ f_{j} \circ T_{j}^{-1}$, we may assume that:

- $\Omega, \Omega_{j}, \hat{\Omega}, \hat{\Omega}_{j}$ are bounded strongly pseudoconvex domains with $C^{k+1, \alpha}$ boundaries such that $\Omega_{j}$ (and $\hat{\Omega}_{j}$, respectively) converges to $\Omega$ (and to $\hat{\Omega}$, respectively) in the $C^{k+1, \alpha}$ topology. More precisely, there exist a neighborhood $U$ (and $\hat{U}$, respectively) of $\operatorname{cl}(\Omega)$ (and of $\operatorname{cl}(\widehat{\Omega})$, respectively) and diffeomorphisms $F_{j}: \operatorname{cl}(\Omega) \rightarrow \operatorname{cl}\left(\Omega_{j}\right)$ and $\widehat{F}_{j}: \operatorname{cl}(\widehat{\Omega}) \rightarrow \operatorname{cl}\left(\hat{\Omega}_{j}\right)$ such that $F_{j}(0)=\widehat{F}_{j}(0)=0$ and the maps $F_{j}, F_{j}^{-1}, \widehat{F}_{j}$ and $\hat{F}_{j}^{-1}$ converge to the identity map in the $C^{k+1, \alpha}$ sense.

- $\rho, \rho_{j}=\rho \circ F_{j}^{-1}, \hat{\rho}, \hat{\rho}_{j}=\hat{\rho} \circ \hat{F}_{j}^{-1}$ are defining functions of $\Omega, \Omega_{j}, \hat{\Omega}, \hat{\Omega}_{j}$, respectively, such that $\left\|\rho-\rho_{j}\right\|_{C^{k+1, \beta}(U)} \rightarrow 0$ and $\left\|\hat{\rho}-\hat{\rho}_{j}\right\|_{C^{k+1, \beta}(\hat{U})} \rightarrow 0$ as $j \rightarrow \infty$ and

$$
\begin{aligned}
(1,0, \ldots, 0) & =\left(\frac{\partial \rho}{\partial z_{1}}(0), \ldots, \frac{\partial \rho}{\partial z_{n}}(0)\right)=\left(\frac{\partial \rho_{j}}{\partial z_{1}}(0), \ldots, \frac{\partial \rho_{j}}{\partial z_{n}}(0)\right) \\
& =\left(\frac{\partial \hat{\rho}}{\partial z_{1}}(0), \ldots, \frac{\partial \hat{\rho}}{\partial z_{n}}(0)\right)=\left(\frac{\partial \hat{\rho}_{j}}{\partial z_{1}}(0), \ldots, \frac{\partial \hat{\rho}_{j}}{\partial z_{n}}(0)\right) .
\end{aligned}
$$

- There exist biholomorphisms $f_{j}: \Omega_{j} \rightarrow \hat{\Omega_{j}}, f: \Omega \rightarrow \widehat{\Omega}$ and a sequence $x_{j} \in \Omega_{j}$ converging to $0 \in \partial \Omega$ as $j \rightarrow \infty$ such that $f_{j}$ converges to $f$ uniformly on every compact subset $K$ of $\Omega$ while $\left|D^{\ell} f_{j}\left(x_{j}\right)\right| \rightarrow \infty$ as $j \rightarrow \infty$ for some multiindex $\ell$ with $1 \leq|\ell| \leq k$. 
Step 2. Simultaneous convexification. This step is directly from [Fornaess 1976]. To the expansion of $\rho$ at 0 ,

$$
\rho(z)=2 \operatorname{Re} z_{1}+\operatorname{Re} \sum \frac{\partial^{2} \rho}{\partial z_{i} z_{j}}(0) z_{i} z_{j}+\frac{1}{2} \sum_{i, j} \frac{\partial^{2} \rho}{\partial z_{i} \bar{z}_{j}}(0) z_{i} \bar{z}_{j}+o\left(|z|^{2}\right),
$$

apply the local biholomorphic change $\Upsilon=\left(w_{1}, w_{2}, \ldots, w_{n}\right)$ of holomorphic coordinate system at the origin 0 defined by

$$
w_{i}(z)=\left\{\begin{aligned}
2 z_{1}+\sum \frac{\partial^{2} \rho}{\partial z_{i} z_{j}}(0) z_{i} z_{j}, & i=1, \\
z_{i}, & i=2, \ldots, n .
\end{aligned}\right.
$$

The new defining function (we continue to use $\rho$, as there is little danger of confusion) takes the form

$$
\rho=\operatorname{Re} w_{1}+\frac{1}{2} \sum_{i, j} \frac{\partial^{2} \rho}{\partial w_{i} \bar{w}_{j}}(0) w_{i} \bar{w}_{j}+\varepsilon(w),
$$

where $\varepsilon(w)=o\left(|w|^{2}\right)$. Note that $\Upsilon(\Omega)$ is strictly convex in a small neighborhood of 0 . Furthermore, there exists a positive integer $N$ such that $\Upsilon\left(U^{\prime} \cap \Omega_{j}\right)$ is strictly convex for any $j>N$. Let $\rho_{j}$ denote $\tilde{\rho}_{j} \circ \Upsilon$, where $\tilde{\rho}_{j}$ is strictly convex on $V^{\prime} \cap \Omega_{j}$ for all $j>N$. Set

$$
\tilde{\rho}(z)=\operatorname{Re} z_{1}+\frac{1}{2} \sum_{i, j} \frac{\partial^{2} \rho}{\partial z_{i} \bar{z}_{j}}(0) z_{i} \bar{z}_{j}+\sigma(z) .
$$

There exists a positive constant $R$ sufficiently large so that the real Hessian forms of $\tilde{\rho}(z)-|z|^{2} /(2 R)-\operatorname{Re} z_{1}$ and $\tilde{\rho}_{j}(z)-|z|^{2} /(2 R)-\operatorname{Re} z_{1}$ are positive definite at every $z \in V^{\prime}$. Choose $h \in C^{\infty}(\mathbb{R})$ such that

$$
\begin{aligned}
h(x)=0 & \text { if } & x \geq 1, \\
0 \leq h(x) \leq 1 & \text { if } & 0 \leq x \leq 1, \\
h(x)=1 & \text { if } & x \leq 0 .
\end{aligned}
$$

Taking a larger value for $N$ if necessary, we may have that the real Hessian forms of

$$
\begin{aligned}
& \operatorname{Re} z_{1}+\frac{|z|^{2}}{2 R}+\frac{1}{N} h\left(\frac{|z|-\eta}{\eta}\right)\left(\tilde{\rho}(z)-\frac{|z|^{2}}{2 R}-\operatorname{Re} z_{1}\right), \\
& \operatorname{Re} z_{1}+\frac{|z|^{2}}{2 R}+\frac{1}{N} h\left(\frac{|z|-\eta}{\eta}\right)\left(\tilde{\rho}_{j}(z)-\frac{|z|^{2}}{2 R}-\operatorname{Re} z_{1}\right)
\end{aligned}
$$

are both positive definite real Hessian at every point of $V_{\delta}:=\left\{z \in \mathbb{C}^{n}:|z|<\delta\right\} \Subset V^{\prime}$ whenever $\eta$ satisfies $0<\eta<\frac{\delta}{3}$. Take $\eta>0$ such that $2^{2 N+2} \eta<\frac{\delta}{3}$ and set 


$$
\begin{gathered}
\tau(z)=\operatorname{Re} z_{1}+\frac{|z|^{2}}{2 R}+\frac{1}{N} \sum_{m=1}^{N} h\left(\frac{|z|-2^{2 m} \eta}{2^{2 m} \eta}\right)\left(\tilde{\rho}(z)-\frac{|z|^{2}}{2 R}-\operatorname{Re} z_{1}\right), \\
\tau_{j}(z)=\operatorname{Re} z_{1}+\frac{|z|^{2}}{2 R}+\frac{1}{N} \sum_{m=1}^{N} h\left(\frac{|z|-2^{2 m} \eta}{2^{2 m} \eta}\right)\left(\tilde{\rho}_{j}(z)-\frac{|z|^{2}}{2 R}-\operatorname{Re} z_{1}\right) .
\end{gathered}
$$

We further let $C=\left\{z \in \mathbb{C}^{n}: \tau(z)<0\right\}, C_{j}=\left\{z \in \mathbb{C}^{n}: \tau_{j}(z)<0\right\}$ and $U^{\prime \prime}=$ $W^{-1}\left(V_{\delta / 3}\right)$. Then $C, C_{j}$ are bounded strictly convex domains such that the restricted mappings

$$
\left.\Upsilon\right|_{U^{\prime \prime} \cap \Omega}: U^{\prime \prime} \cap \Omega \rightarrow V_{\delta / 3} \cap C \quad \text { and }\left.\quad \Upsilon\right|_{U^{\prime \prime} \cap \Omega_{j}}: U^{\prime \prime} \cap \Omega_{j} \rightarrow V_{\delta / 3} \cap C_{j}
$$

are biholomorphisms, and $\tau_{j}$ converges to $\tau$ in the $C^{k+1, \beta}$ norm, for every $\beta$, $0<\beta<\alpha$.

Apply the same process to $\widehat{\Omega}$ and to $\hat{\Omega}_{j}$ at 0 . Denote by $\widehat{C}, \widehat{C}_{j}$ the respective strictly convex domains with defining functions $\hat{\tau}, \widehat{\tau_{j}}$ and $\widehat{W}: \widehat{U} \rightarrow \widehat{V}$ produced by the same procedures.

Step 3. Estimates. Let $\omega \in C \cap V^{\prime} \cap\left(\bigcap_{j=1}^{\infty} C_{j}\right)$ be a point that admits an extremal $\overline{\operatorname{map} e}: \operatorname{cl}(\Delta) \rightarrow \operatorname{cl}(C)$ satisfying

$$
e(0)=\omega, \quad e(1)=0, \quad \text { and } \quad e(\operatorname{cl}(\Delta)) \subset \operatorname{cl}(C) \cap V^{\prime} .
$$

Let $e^{\prime}(0)=\mu v$ where $|v|=1$. Let $L: \operatorname{cl}\left(\mathbb{B}^{n}\right) \rightarrow \operatorname{cl}(C)\left(L_{j}: \operatorname{cl}\left(\mathbb{B}^{n}\right) \rightarrow \operatorname{cl}\left(C_{j}\right)\right.$, respectively) be the Lempert representative map of $C\left(C_{j}\right.$, respectively) at $\omega$. By Proposition 4.2, there exists a $\epsilon>0$ such that $\lim _{j \rightarrow \infty}\left\|L_{j}-L\right\|_{C^{k, \beta}\left(\mathrm{cl}\left(\mathbb{B}_{\varepsilon}^{n}\right)\right)}=0$ for any $\beta$ with $0<\beta<\alpha$. Let $\Gamma$ be a closed cone containing $v$ in $\operatorname{cl}\left(\mathbb{B}^{n}\right)$ so that $L(\Gamma) \subset \operatorname{cl}(C) \cap V_{\delta / 3}$ and $L_{j}(\Gamma) \subset \operatorname{cl}\left(C_{j}\right) \cap V_{\delta / 3}$ for all $j>N$. Let

$$
\Upsilon^{-1}(\omega)=\zeta, \quad f(\zeta)=\hat{\zeta}, \quad f_{j}(\zeta)=\hat{\zeta}_{j}, \quad \hat{\Upsilon}(\widehat{\zeta})=\widehat{\omega}, \quad \hat{\Upsilon}\left(\widehat{\zeta}_{j}\right)=\widehat{\omega}_{j}
$$

and let $\widehat{L}: \operatorname{cl}\left(\mathbb{B}^{n}\right) \rightarrow \operatorname{cl}(\widehat{C})$ and $\widehat{L}_{j}: \operatorname{cl}\left(\mathbb{B}^{n}\right) \rightarrow \operatorname{cl}\left(\widehat{C}_{j}\right)$, respectively, denote the Lempert representative map of $\widehat{C}$ at the point $\widehat{\omega}$ and the Lempert representative map of $\widehat{C}_{j}$ at the point $\widehat{\omega}_{j}$.

Consider now the composite maps $\hat{L}^{-1} \circ \hat{\Upsilon} \circ f \circ \Upsilon^{-1} \circ L: \Gamma \rightarrow \mathbb{B}^{n}$ and ${\hat{L_{j}}}^{-1} \circ \hat{\Upsilon} \circ f_{j} \circ \Upsilon^{-1} \circ L_{j}: \Gamma \rightarrow \mathbb{B}^{n}$. Denote by $h: \operatorname{cl}(D) \rightarrow \operatorname{cl}(C)$ the extremal map satisfying $h(0)=\omega, h^{\prime}(0)=\lambda \zeta$, for some $\lambda>0$, and by $\hat{h}=\widehat{\Upsilon} \circ f \circ \Upsilon^{-1} \circ h$ : $\operatorname{cl}(D) \rightarrow \operatorname{cl}(\widehat{C})$ the extremal map satisfying

$$
\hat{h}(0)=\widehat{\omega}, \quad \hat{h}^{\prime}(0)=\hat{\lambda}|\zeta| \frac{\left.d\left(\hat{\Upsilon} \circ f \circ \Upsilon^{-1}\right)\right|_{\omega}(\zeta)}{\mid d\left(\hat{\Upsilon} \circ f \circ \Upsilon^{-1}|\omega(\zeta)|\right.}
$$

for some $\hat{\lambda}$. Since $\hat{C}$ is strictly convex and $f$ extends to $\operatorname{cl}(\Omega)$ as a $C^{k, \gamma}$ diffeomorphism for all $\gamma<\alpha$, we have 


$$
\widehat{W}^{-1} \circ \widehat{L}\left(\frac{\left.|\zeta| d\left(\hat{W} \circ f \circ W^{-1}\right)\right|_{\omega}(\zeta)}{\mid d\left(\left.\hat{W} \circ f \circ W^{-1}\right|_{\omega}(\zeta) \mid\right.}\right)=f \circ W^{-1} \circ L(\zeta) .
$$

By the same reasoning we also have

$$
\widehat{W}_{j}^{-1} \circ \hat{L}\left(\frac{\left.|\zeta| d\left(\hat{W} \circ f_{j} \circ W^{-1}\right)\right|_{\omega}(\zeta)}{\mid d\left(\left.\hat{W} \circ f_{j} \circ W^{-1}\right|_{\omega}(\zeta) \mid\right.}\right)=f_{j} \circ W^{-1} \circ L_{j}(\zeta) .
$$

Considering the left-hand sides of the preceding identities, for any $\beta, 0<\beta<\alpha$, we obtain

$$
\lim _{j \rightarrow \infty}\left\|f \circ W^{-1} \circ L-f_{j} \circ W^{-1} \circ L_{j}\right\|_{C^{k, \beta}\left(\Gamma_{\varepsilon}\right)}=0,
$$

where $\Gamma_{\varepsilon}=\Gamma \backslash\{z \in \Gamma:|z|<\varepsilon\}$. Therefore

$$
\begin{aligned}
\lim _{j \rightarrow \infty}\left\|f \circ \Upsilon^{-1} \circ L-f \circ F_{j}^{-1} \circ \Upsilon^{-1} \circ L_{j}\right\|_{C^{k, \beta}\left(\Gamma_{\varepsilon}\right)} \\
\quad=\lim _{j \rightarrow \infty}\left\|f \circ F_{j}^{-1} \circ \Upsilon^{-1} \circ L_{j}-f_{j} \circ \Upsilon^{-1} \circ L_{j}\right\|_{C^{k, \beta}\left(\Gamma_{\varepsilon}\right)} .
\end{aligned}
$$

Hence

$$
\begin{aligned}
&\left\|f \circ \Upsilon^{-1} \circ L-f \circ F_{j}^{-1} \circ \Upsilon^{-1} \circ L_{j}\right\|_{C^{k, \beta}\left(\Gamma_{\varepsilon}\right)} \\
& \lesssim\left\|\Upsilon^{-1} \circ L-F_{j}^{-1} \circ \Upsilon^{-1} \circ L_{j}\right\|_{C^{k, \beta}\left(\Gamma_{\varepsilon}\right)} \\
& \lesssim\left\|\Upsilon^{-1} \circ L-\Upsilon^{-1} \circ L_{j}\right\|_{C^{k, \beta}\left(\Gamma_{\varepsilon}\right)}+\left\|\Upsilon^{-1} \circ L_{j}-F_{j}^{-1} \circ \Upsilon^{-1} \circ L_{j}\right\|_{C^{k, \beta}\left(\Gamma_{\varepsilon}\right)} \\
& \lesssim\left\|L-L_{j}\right\|_{C^{k, \beta}\left(\Gamma_{\varepsilon}\right)}+\left\|\left(\mathrm{id}-F_{j}^{-1}\right) \Upsilon^{-1} \circ L_{j}\right\|_{C^{k, \beta}\left(\Gamma_{\varepsilon}\right)} \rightarrow 0 \text { as } j \rightarrow \infty .
\end{aligned}
$$

On the other hand, by the proof argument of Lemma 4.5, we have

$\left\|f \circ F_{j}^{-1} \circ \Upsilon^{-1} \circ L_{j}-f_{j} \circ \Upsilon^{-1} \circ L_{j}\right\|_{C^{k, \beta}\left(\Gamma_{\varepsilon}\right)}$

$$
=\left\|\left(f-f_{j} \circ F_{j}\right) \circ F_{j}^{-1} \circ \Upsilon^{-1} \circ L_{j}\right\|_{C^{k, \beta}\left(\Gamma_{\varepsilon}\right)} \gtrsim\left\|\left(f-f_{j} \circ F_{j}\right)\right\|_{C^{k, \beta}(\sigma)}
$$

on a sufficiently small neighborhood $\sigma$ of $p$. This contradicts (1).

To complete the proof let us now suppose that (2) holds. If $\left|x_{j}-y_{j}\right|>\kappa$ for some positive constant $\kappa$, then

$$
\frac{\left|D^{\gamma} f_{j}\left(x_{j}\right)-D^{\gamma} f_{j}\left(y_{j}\right)\right|}{\left|x_{j}-y_{j}\right|^{\beta}}<\frac{2 C}{\kappa^{\beta}}
$$

holds for some constant $C$. Without loss of generality, we may assume that $x_{j} \rightarrow$ $p \in \partial \Omega$ and $\left|x_{j}-y_{j}\right|<\kappa$. Suppose that there exist sequences $x_{j}, y_{j} \in \Omega_{j}$ and a positive constant $v$ such that $x_{j} \rightarrow 0 \in \partial \Omega$ as $j \rightarrow \infty$ and $\left|x_{j}-y_{j}\right|<v$ so that

$$
\frac{\left|D^{\ell} f_{j}\left(x_{j}\right)-D^{\ell} f_{j}\left(y_{j}\right)\right|}{\left|x_{j}-y_{j}\right|^{\beta}} \rightarrow \infty
$$


as $j \rightarrow \infty$ for some multiindex $\ell$ where $|\ell|=k$. Repeating Steps 1, 2 and 3 above, we again arrive at a contradiction. Hence the proof of Lemma 4.7 is complete.

Proof of Theorem 4.3. Throughout the proof, we shall take subsequences from the $\left\{f_{j}\right\}$ several times. But we denote them by the same notation $f_{j}$, since there is little danger of any confusion.

By Cauchy estimates and the standard normal family theorem, for any compact subset $K$ of $\Omega$ we have

$$
\lim _{j \rightarrow \infty}\left\|f_{j}-f\right\|_{C^{k, \beta}(K)}=0 .
$$

Denote by $K_{\eta}=\{z \in \Omega \mid \operatorname{dist}(\partial \Omega, z) \geq \eta\}$. Then there exist $N>0$ and $\eta>0$ such that $F_{j}(K) \Subset K_{\eta} \Subset \Omega$ for all $j>N$. So

$$
\left\|f_{j} \circ F_{j}-f\right\|_{C^{k, \beta}(K)} \leq\left\|f_{j} \circ F_{j}-f_{j}\right\|_{C^{k, \beta}(K)}+\left\|f_{j}-f\right\|_{C^{k, \beta}(K)} \rightarrow 0
$$

as $j \rightarrow \infty$ for all $\beta<\alpha$ by the proof of Lemma 4.6.

Let $\lambda>0$. For $x \in \operatorname{cl}(\Omega)-K_{\epsilon}$, there exists $y \in K_{\epsilon}$ such that $|x-y|<\epsilon$. By Lemma 4.7, we have

$$
\begin{aligned}
\left|D^{l}\left(f_{j} \circ F_{j}\right)(x)-D^{l} f(x)\right| \leq & \left|D^{l}\left(f_{j} \circ F_{j}\right)(x)-D^{l}\left(f_{j} \circ F_{j}\right)(y)\right| \\
& +\left|D^{l}\left(f_{j} \circ F_{j}\right)(y)-D^{l} f(y)\right|+\left|D^{l} f(y)-D^{l} f(x)\right| \\
\lesssim & 2|x-y|^{\beta}+\epsilon \lesssim 2 \epsilon^{\beta}+\epsilon .
\end{aligned}
$$

Since

$$
\begin{aligned}
& \sup _{\substack{x \in \mathrm{c}(\Omega) \\
0 \leq|\ell| \leq k}}\left|D^{\ell}\left(f_{j} \circ F_{j}\right)(x)-D^{\ell} f(x)\right| \\
& \leq \max \left\{\sup _{\substack{x \in K_{\epsilon} \\
0 \leq|\ell| \leq k}}\left|D^{\ell}\left(f_{j} \circ F_{j}\right)(x)-D^{\ell} f(x)\right|, \sup _{\substack{x \in \mathrm{cl}(\Omega) \backslash K_{\epsilon} \\
0 \leq|\ell| \leq k}}\left|D^{\ell}\left(f_{j} \circ F_{j}\right)(x)-D^{\ell} f(x)\right|\right\},
\end{aligned}
$$

there exist $N>0$ and $\epsilon$ such that, for all $j>N$,

$$
\sup _{\substack{x \in \operatorname{cl}(\Omega) \\ 0 \leq|\ell| \leq k}}\left|D^{\ell}\left(f_{j} \circ F_{j}\right)(x)-D^{\ell} f(x)\right|<\lambda .
$$

Let $\delta_{\ell}(x, y):=\frac{\left|D^{\ell}\left(f_{j} \circ F_{j}\right)(x)-D^{\ell} f(x)-D^{\ell}\left(f_{j} \circ F_{j}\right)(y)+D^{\ell} f(y)\right|}{|x-y|^{\beta}}$. Then

$$
\sup _{\substack{x, y \in \operatorname{cl}(\Omega) \\|\ell|=k}} \delta_{\ell}(x, y) \leq \max \left(\sup _{\substack{x \in \operatorname{cl}(\Omega) \\ y \in K_{\epsilon},|\ell|=k}} \delta_{\ell}(x, y), \sup _{\substack{x, y \in \operatorname{cl}(\Omega) \backslash K_{\epsilon} \\|\ell|=k}} \delta_{\ell}(x, y)\right) .
$$


Consider the first supremum in the right-hand side of (1). For $x \in \operatorname{cl}(\Omega), y \in K_{\epsilon}$, there exists $z \in K_{\epsilon}$ such that $\operatorname{dist}\left(K_{\epsilon}, x\right)=|x-z|$. Therefore we see that

$$
\begin{aligned}
\delta_{\ell}(x, y) \leq & \frac{\left|D^{\ell}\left(f_{j} \circ F_{j}\right)(x)-D^{\ell} f(x)-D^{\ell}\left(f_{j} \circ F_{j}\right)(z)+D^{\ell} f(z)\right|}{|x-y|^{\beta}} \\
& +\frac{\left|D^{\ell}\left(f_{j} \circ F_{j}\right)(z)-D^{\ell} f(z)-D^{\ell}\left(f_{j} \circ F_{j}\right)(y)+D^{\ell} f(y)\right|}{|x-y|^{\beta}} \\
& \lesssim \delta_{\ell}(x, z)+\delta_{\ell}(z, y),
\end{aligned}
$$

because $|x-y| \geq|x-z|$ and $|y-z| \leq|y-x|+|x-z| \leq 2|x-y|$. Notice now that, for $\mu$ satisfying $\beta+\mu<\alpha$, we have that $\delta_{\ell}(x, z) \lesssim|x-z|^{\mu}<\epsilon^{\mu}$. So

$$
\sup _{\substack{x \in \mathrm{cl}(\Omega), y \in K_{\epsilon} \\|\ell|=k}} \delta_{\ell}(x, y)<\lambda
$$

for any $j>N$. (For this last, one may need to adjust the sizes of $N$ and $\epsilon$.)

Consider now the second supremum in the right-hand side of (1). Let $x, y \in$ $\operatorname{cl}(\Omega)-K_{\epsilon}$. If $|x-y|<\epsilon$, then for $\mu$ satisfying $\beta+\mu<\alpha, \delta_{\ell}(x, y) \lesssim|x-y|^{\mu}<\epsilon^{\mu}$. If $|x-y| \geq \epsilon$, let $z$ be a point in $K_{\epsilon}$ satisfying $|x-z|=\operatorname{dist}\left(K_{\epsilon}, x\right)$. Then $\delta_{\ell}(x, y) \lesssim \delta_{\ell}(x, z)+\delta_{\ell}(z, y)$, since $|x-z|<\epsilon<|x-y|$ and $|y-z|<2|x-y|$. So

$$
\sup _{\substack{x, y \in \operatorname{cl}(\Omega) \backslash K_{\epsilon} \\|\ell|=k}} \delta_{l}(x, y)<\lambda .
$$

Since $\lambda>0$ is arbitrary, we see that

$$
\lim _{j \rightarrow \infty} \sup _{\substack{x, y \in \operatorname{cl}(\Omega) \\|\ell|=k}} \delta_{\ell}(x, y)=0
$$

for any $\beta<\alpha$. This completes the proof of Theorem 4.3.

\section{Conjugation by diffeomorphism}

For isometries of compact Riemannian manifolds, semicontinuity involves not just that nearby metrics have isometry groups which are isomorphic to subgroups of the unperturbed metric, but that the isomorphisms are obtainable via conjugation by diffeomorphism (cf. [Ebin 1968; Guillemin et al. 2002]). This conjugation by diffeomorphism actually applies in the case of bounded $C^{\infty}$ strongly pseudoconvex domains as well; see, e.g., [Greene and Krantz 1982b; Greene et al. 2011]. Naturally, the $C^{\infty}$ hypothesis used in these references is, as usually happens, replaceable by a finite differentiability hypotheses simply by tracing through the arguments and checking how many derivatives are needed. 
In this section, the subject will be investigated of the finite differentiability version of the conjugation by diffeomorphism results already shown in the references indicated in the $C^{\infty}$ case. These results are of active interest because, by this time, quite precise results are known about extension to the boundary with finite smoothness of automorphisms of bounded strongly pseudoconvex domains with boundaries of finite smoothness. In particular, the results of the previous sections give motivation to study the issues discussed in the present section.

In the $C^{\infty}$ version presented in [Greene and Krantz 1982a] and [Greene et al. 2011], the basic technique was to pass to the double in the topologist's sense of the domain, thus creating a situation to which the compact manifold results could be applied. This technique can still be applied in the present case. The difference is that we need now to keep track of how many derivatives are lost in the passage to the double. For the manifold with boundary itself, no derivatives are lost. It is shown in [Munkres 1963] that a $C^{k}$ manifold with boundary, $k \geq 1$, has a $C^{k}$ double that is unique up to $C^{k}$ diffeomorphism.

In our case Theorem 4.3 allows us to have the $C^{k, \alpha}$ metric double for every $k \geq 2$ and any $0<\alpha<1$. But, the need to make the group act on the double requires that the doubling construction be invariant under the group, which actually needs $k \geq 4$. And this will turn out to reduce the guaranteed differentiability of the conjugating diffeomorphism.

To facilitate the discussion, we introduce a definition (similar to one given in Section 2) of the sense in which a sequence of groups of diffeomorphisms might converge to a limit group:

Suppose that $M$ is a compact $C^{k}$ manifold with boundary, $k$ a positive integer. Suppose that $G_{0}$ is a compact Lie group of $C^{k}$ diffeomorphisms of $M$ and that moreover $G_{j}, j=1,2, \ldots$ are a sequence of compact Lie groups of $C^{k}$ diffeomorphisms. Then we say that the sequence $G_{j}$ converges to $G_{0}$ in the $C^{k}$ sense if for each $\epsilon>0$ there is a number $j_{0}$ such that, if $j>j_{0}$ and $g \in G_{j}$, then there is an element $g_{0} \in G_{0}$ such that the distance from $g$ to $g_{0}$ is less than $\epsilon$. Here the distance means relative to any metric on the set of $C^{k}$ mappings which gives the usual $C^{k}$ topology on $C^{k}$ maps from $M$ to $M$.

In these terms, we can now formulate the general real-differentiable result we shall use in the complex case:

Theorem 5.1. Suppose that $M$ is a compact $C^{r}$ manifold with boundary and that $r>2$ is an integer, that $G_{0}$ is a compact Lie group of $C^{r}$ diffeomorphisms of $M$, and that $G_{j}, j=1,2, \ldots$, is a sequence of compact groups of $C^{r}$ diffeomorphisms which converge in the $C^{r}$ sense to $G_{0}$. Then, for all $j$ sufficiently large, there is a $C^{r-2}$ diffeomorphism $F_{j}$ of $M$ to itself such that $F_{j} \circ G_{j} \circ F_{j}^{-1}$ is a subgroup of $G_{0}$, i.e., $F_{j}$ conjugates the elements of $G_{j}$ into elements of $G_{0}$. 
The proof of this theorem follows almost precisely the pattern of the proof of Theorem 0.1 in [Greene and Krantz 1982a] (cf. [Greene et al. 2011, Theorem 4.4.1]). The only difference is that we must here keep some track of the number of derivatives involved: Ebin's theorem concerned the $C^{\infty}$ case so that loss of a derivative or two or indeed of any finite number was irrelevant. This is why we need the results of Section 4.

Discussion of the proof of Theorem 5.1. As in Section 2, the essential method is to pass to the double of $M$ and extend the action of the groups to the double. Then one can use Ebin's result in the form presented in [Guillemin et al. 2002], where only $C^{1}$ is required for the closeness of the group actions. But here we have to keep track of degrees of differentiability as opposed to the $C^{\infty}$ situation of Section 2.

The most natural way to form the equivariant double is via metric construction as already explained in Section 2 (cf. [Greene and Krantz 1982a]). As before one takes a metric $g$ on the manifold with boundary that is invariant under the group $G$. Then one defines charts in neighborhoods of boundary points $p$ using the normal field to the boundary. Specifically, let $N(q)$ be the $g$-metric normal to the tangent space to the boundary $\partial M$ at the point $q$ in $\partial M$. Then one defines charts in a neighborhood of points $p$ in the boundary as follows: map $\partial M \times(-\epsilon, \epsilon) \rightarrow M$ by $(q, t) \mapsto \exp _{q}(t N(q))$, where exp is the geodesic exponential map of the Riemannian metric $g$ and $N(q)$ is the inward pointing normal at $q$. Choosing a chart around $p$ in $\partial M$ then gives a chart in a neighborhood of $p$ in the double of $M$ if we interpret $\exp _{q}(t N(q))$ to be in the second copy of $M$ when $t<0$.

In terms of derivative loss, the choice of the normal vector $N$ loses one derivative, since it is an algebraic process using $g$ and the tangent space of the boundary and the latter is not $C^{r}$ but $C^{r-1}$. But an additional loss of derivative, so that two derivatives are lost, occurs because the exponential map is defined by the geodesic equation and that equation involves the Christoffel symbols, which involve the first derivative of the metric $g$. And the metric $g$ has already lost one derivative in the averaging over the action of the group $G$.

Thus one obtains a $G$-equivariant construction of the double $\tilde{M}$ of $M$ and by construction the action of $G$ on $M$ extends to be an action of $G$ on $\tilde{M}$. This extended group action is $C^{r-2}$. Associate to the group $G$ a group $\widetilde{G}$ defined to be $G \oplus \mathbb{Z}_{2}$. Then $\widetilde{G}$ acts on $\tilde{M}$ in a natural way. Namely, we label the elements of $\tilde{M}$ by $(m, a)$ where $m \in M$ and $a \in\{0,1\}$ with 0 corresponding to the original of $M$ and 1 corresponding to the second copy of $M$. Then we let $(g, b)$ acting on $(m, a)$ be

$$
(g(m), a+b),
$$

where the addition $a+b$ is in $\mathbb{Z}_{2}$. For example $\left(\operatorname{id}_{G}, 1\right)$ acts on $\tilde{M}$ as the "flip" map that interchanges the two copies of $M$. 
Note that the fixed point set of $\left(\operatorname{id}_{G}, 1\right)$ is exactly $\partial M$. And, for any element $g \in G$, the fixed point set of $(g, 1)$ is contained in $\partial M$, though it need not be all of it, and can indeed be empty if the action of $g$ on $\partial M$ has no fixed point. These observations will be important later.

Now we turn to the explicit situation of Theorem 5.1. We choose a sequence of $G_{j}$-invariant $C^{r-1}$ metrics on $M$, which can clearly be taken to converge in the $C^{r-1}$ sense to a $G_{0}$-invariant $C^{r-1}$ metric on $M$. Passing to the double $\widetilde{M}$ gives a sequence of $\widetilde{G}_{j}$ group actions on $\tilde{M}$. We can form a sequence of $\widetilde{G}_{j}$ invariant metrics by combining, via a partition of unity, a product metric structure near the boundary with the $G_{j}$-invariant metric on the interior of $M$. Namely, as similar to before, let $E_{j}$ be the exponential map of the metric $g_{j}, j=0,1,2, \ldots$, acting on the normal bundle of the boundary $\partial M$ of $M$ in $M$ to give maps also to be denoted by $E_{j}: \partial M \times(-a, a) \rightarrow \tilde{M}$ of the boundary $\partial M$ of $M$ producted with an open interval $(-a, a)$ into $\tilde{M}$. The size of $a$ can, by the $C^{r-2}$ convergence of the $E_{j}$ to $E_{0}$, be chosen uniformly so that these $E_{j}$ are diffeomorphisms onto their images in $\partial M$, which themselves converge in the $C^{r-2}$ sense to the limit $C^{r-2}$ diffeomorphism $E_{0}$.

Via this diffeomorphism, we transfer the product metrics on $\partial M \times[0, \epsilon)$, namely $H_{j} \times d t^{2}$, to the associated tubular neighborhoods of $\partial M$ in $M$. This transfer gives a $\widetilde{G}_{j}$-invariant metric for each $j$ and these metrics converge $C^{r-2}$ to the limit $\widetilde{G}_{0}$-invariant metric. Now we can combine, using a $\widetilde{G}_{j}$-equivariant partition of unity, these product metrics with the $G_{j}$-invariant metric $g_{j}$ on $M$ to obtain a $\widetilde{G}_{j}$-invariant metric on $\tilde{M}$, to be denoted $\widetilde{g}_{j}$. This metric is $C^{r-2}$. And it converges in the $C^{r-2}$ topology to the corresponding $\widetilde{G}_{0}$-invariant metric $\widetilde{g}_{0}$ on $\tilde{M}$. (The $G_{j}$-equivariant partition of unity is obtained by taking the partition of unity function to depend on $t$ alone, $t$ as above).

Now we can apply Ebin's theorem, in the form given in [Guillemin et al. 2002] and [Kim 1987], for the $C^{r-2}$ case to get $C^{r-2}$ diffeomorphisms $F_{j}: \tilde{M} \rightarrow \tilde{M}$ which conjugate $\widetilde{G}_{j}$ into a subgroup of $\widetilde{G}_{0}$. (Here we are reasoning as follows: there is a diffeomorphism that conjugates $\operatorname{Isom}\left(\widetilde{g}_{j}\right)$ into a $\operatorname{subgroup}$ of $\operatorname{Isom}\left(\widetilde{g}_{0}\right)$ and hence conjugates $\widetilde{G}_{j}$ into a subgroup of $\operatorname{Isom}\left(\widetilde{g}_{0}\right)$ and these diffeomorphisms can be taken to converge to the identity map. So the image of $\widetilde{G}_{j}$ under this conjugation is close to $\widetilde{G}_{0}$ for large $j$ in the sense of $C^{r-2}$ convergence. By the classical theorem of [Montgomery and Samelson 1943], this conjugation image is in fact itself conjugate in $\operatorname{Isom}\left(\widetilde{g}_{0}\right)$ to a subgroup of $G_{0}$ by an element close to the identity. (See, e.g., [Greene et al. 2011, Chapter 4], for more detail.)

Now we need to know that in fact the conjugation image of $G_{j}$ lies in $G_{0}$, not just in $\widetilde{G}_{0}$. For this, we need only show that the diffeomorphism that is conjugating takes $\partial M$ to itself. This can be deduced as follows: let us denote by $\operatorname{Fix}(\psi)$ the fixed point set of $\psi$. Then conjugation takes fixed points to fixed points in the 
sense that $\operatorname{Fix}\left(f \circ \psi \circ f^{-1}\right)=f(\operatorname{Fix}(\psi))$. Now consider the case of $\psi$ equal to the flip map which interchanges the two copies of $M$ in $\tilde{M}$. When $f$ is close to the identity, $f \circ \psi \circ f^{-1}$ has to belong to the part of the group that interchanges the two components. So its fixed point set cannot be larger than $\partial M$. Thus $f(\partial M)$ lies in $\partial M$ and hence equals $\partial M$ (since $f$ is a diffeomorphism of $\partial M$ onto its image).

This completes the proof of the theorem.

Note that these considerations of fixed points of the interchange map did not arise in Section 2, since we were concerned there only with isomorphism, not with the existence of a conjugating diffeomorphism of the manifolds with boundary.

The application to the strongly pseudoconvex case now follows:

Theorem 5.2. Let $\Omega_{0}$ be a bounded strongly pseudoconvex domain with a $C^{k, \alpha}$ boundary in $\mathbb{C}^{n}$, not biholomorphic to the unit ball. Then there is a $C^{k, \alpha}$ neighborhood $\mathcal{N}$ of $\Omega_{0}$ such that, for any $\Omega \in \mathcal{N}$, there is a $C^{k-3}$ diffeomorphism $f: \Omega \rightarrow \Omega_{0}$ with the property that $f \circ \operatorname{Aut}(\Omega) \circ f^{-1} \subset \operatorname{Aut}\left(\Omega_{0}\right)$.

Theorem 5.2 is derived from Theorem 5.1 by exactly the arguments of [Greene and Krantz 1982a].

In outline, these arguments are as follows: first, the stable estimation of Bergman metric curvature (Theorem 3.1) in Section 3 guarantees that, if $\left\{\Omega_{j}\right\}$ is a sequence of domains converging in $C^{k, \alpha}$ to $\Omega_{0}(k \geq 4)$ with $\Omega_{0}$ not biholomorphic to the ball, and if $p_{0} \in \Omega_{0}$, then there is a $\delta_{p}>0$ such that the distance $\phi_{j}\left(p_{0}\right)$ to $\mathbb{C}^{n}-\Omega_{j}$ is at least $\delta_{p}$ for all $\phi_{j} \in \operatorname{Aut}\left(\Omega_{j}\right)$ for all $j$ sufficiently large. This in turn makes possible the application of normal families arguments to show that for every sequence $\phi_{j} \in \operatorname{Aut}\left(\Omega_{j}\right)$, there is a subsequence $\left\{\phi_{j_{k}}\right\}$ which converges uniformly on compact subsets (of $\Omega_{0}$ ). The uniformity of boundary behavior established in Section 4 then implies that this subsequence converges uniformly in the $C^{k-3}$ topology on the closure of the domains, where comparison over different domains is via fixed diffeomorphisms of $\Omega_{j} \rightarrow \Omega_{j}$ for each $j$, these converging in the $C^{k}$ topology to the identity. Thus one passes to the situation of Theorem 5.1. For further details, the reader can consult [Greene and Krantz 1982a].

\section{Concluding remarks}

Semicontinuity of symmetry in the general sense is an idea with deep roots in intuition to the point that it arguably predates formal mathematical thought altogether. In precise form, when all the symmetry groups belong to one fixed (compact) Lie group, it was given definitive formulation in the result of [Montgomery and Samelson 1943]. The situation for isometry groups and automorphism groups is made more delicate because a priori not all the groups are even isomorphic to subgroups of any fixed Lie group. In [Greene and Krantz 1985], ways of dealing with this issue in the automorphism group case were introduced. The results obtained turned 
out to have some interesting applications, e.g., they played a role in [Bedford and Dadok 1987]. One of the main points of the first part of this paper was that, on account of normal families considerations, in fact this difficulty of the groups not belonging a priori to a fixed larger group is obviated in very general situations. All that is needed is that the groups keep some fixed compact set in the domain (or manifold) within another fixed compact set: this is in effect the stably interior property introduced in Section 2. The remainder of the paper describes how this condition can be guaranteed in the case of $C^{2}$ strongly pseudoconvex domains. In view of the great generality of the stably interior property, it is natural to ask whether some similar guarantee of the property might be available for other classes of domains, for example, those of finite type in the sense of D'Angelo. This would seem to be a potentially fruitful topic for further investigation.

\section{References}

[Bedford and Dadok 1987] E. Bedford and J. Dadok, "Bounded domains with prescribed group of automorphisms”, Comment. Math. Helv. 62:4 (1987), 561-572. MR 89c:32078 Zbl 0647.32027

[Diederich 1973] K. Diederich, "Über die 1. und 2. Ableitungen der Bergmanschen Kernfunktion und ihr Randverhalten”, Math. Ann. 203 (1973), 129-170. MR 48 \#6472 Zbl 0253.32011

[Ebin 1968] D. G. Ebin, “On the space of Riemannian metrics”, Bull. Amer. Math. Soc. 74 (1968), 1001-1003. MR 37 \#6965 Zbl 0172.22905

[Efimov 1995] A. M. Efimov, "A generalization of the Wong-Rosay theorem for the unbounded case”, Mat. Sb. 186:7 (1995), 41-50. In Russian; translated in Sb. Math. 186:7 (1995), 967-976. MR 97d:32030 Zbl 0865.32020

[Epstein 1965] B. Epstein, Orthogonal families of analytic functions, Macmillan, New York, 1965. MR 31 \#4916 Zbl 0145.08901

[Fefferman 1974] C. Fefferman, "The Bergman kernel and biholomorphic mappings of pseudoconvex domains”, Invent. Math. 26 (1974), 1-65. MR 50 \#2562 Zbl 0289.32012

[Fornaess 1976] J. E. Fornaess, "Embedding strictly pseudoconvex domains in convex domains", Amer. J. Math. 98:2 (1976), 529-569. MR 54 \#10669 Zbl 0334.32020

[Gaussier et al. 2002] H. Gaussier, K.-T. Kim, and S. G. Krantz, "A note on the Wong-Rosay theorem in complex manifolds", Complex Var. Theory Appl. 47:9 (2002), 761-768. MR 2003f:32025 Zbl 1044.32019

[Greene and Krantz 1982a] R. E. Greene and S. G. Krantz, "The automorphism groups of strongly pseudoconvex domains”, Math. Ann. 261:4 (1982), 425-446. MR 84c:32032 Zbl 0531.32016

[Greene and Krantz 1982b] R. E. Greene and S. G. Krantz, "Deformation of complex structures, estimates for the $\bar{\partial}$ equation, and stability of the Bergman kernel", Adv. in Math. 43:1 (1982), 1-86. MR 84b:32026 Zbl 0504.32016

[Greene and Krantz 1985] R. E. Greene and S. G. Krantz, "Normal families and the semicontinuity of isometry and automorphism groups", Math. Z. 190:4 (1985), 455-467. MR 87d:32055 Zbl 0584.58014

[Greene and $\mathrm{Wu}$ 1979] R. E. Greene and H. Wu, Function theory on manifolds which possess a pole, Lecture Notes in Mathematics 699, Springer, Berlin, 1979. MR 81a:53002 Zbl 0414.53043 
[Greene et al. 2011] R. E. Greene, K.-T. Kim, and S. G. Krantz, The geometry of complex domains, Progress in Mathematics 291, Birkhäuser, Boston, 2011. MR 2012c:32001 Zbl 1239.32011

[Guillemin et al. 2002] V. Guillemin, V. Ginzburg, and Y. Karshon, Moment maps, cobordisms, and Hamiltonian group actions, Mathematical Surveys and Monographs 98, American Mathematical Society, Providence, RI, 2002. MR 2003m:53149 Zbl 1197.53002

[Kim 1987] Y. W. Kim, "Semicontinuity of compact group actions on compact differentiable manifolds”, Arch. Math. (Basel) 49:5 (1987), 450-455. MR 88m:57046 Zbl 0615.57020

[Kim and Yu 1996] K.-T. Kim and J. Yu, "Boundary behavior of the Bergman curvature in strictly pseudoconvex polyhedral domains”, Pacific J. Math. 176:1 (1996), 141-163. MR 97k:32037 Zbl 0886.32020

[Klembeck 1978] P. F. Klembeck, "Kähler metrics of negative curvature, the Bergmann metric near the boundary, and the Kobayashi metric on smooth bounded strictly pseudoconvex sets", Indiana Univ. Math. J. 27:2 (1978), 275-282. MR 57 \#3455 Zbl 0422.53032

[Krantz 2001] S. G. Krantz, Function theory of several complex variables, AMS Chelsea, Providence, RI, 2001. MR 2002e:32001 Zbl 1087.32001

[Lempert 1981] L. Lempert, "La métrique de Kobayashi et la représentation des domaines sur la boule”, Bull. Soc. Math. France 109:4 (1981), 427-474. MR 84d:32036 Zbl 0492.32025

[Lempert 1986] L. Lempert, "A precise result on the boundary regularity of biholomorphic mappings", Math. Z. 193:4 (1986), 559-579. MR 88a:32033 Zbl 0603.32013

[Lu 1966] Q.-K. Lu, "On Kaehler manifolds with constant curvature”, Acta Math. Sinica 16:2 (1966), 269-281. In Chinese; translated in Chinese Math. Acta 8 (1966), 283-298. MR 34 \#6806 Zbl 0173.33001

[Montgomery and Samelson 1943] D. Montgomery and H. Samelson, "Transformation groups of spheres", Ann. of Math. (2) 44 (1943), 454-470. MR 5,60b Zbl 0063.04077

[Mujica 1986] J. Mujica, Complex analysis in Banach spaces: holomorphic functions and domains of holomorphy in finite and infinite dimensions, North-Holland Mathematics Studies 120, NorthHolland, Amsterdam, 1986. MR 88d:46084 Zbl 0586.46040

[Munkres 1963] J. R. Munkres, Elementary differential topology, Annals of Mathematics Studies 54, Princeton University Press, Princeton, NJ, 1963. MR 29 \#623 Zbl 0107.17201

[Ohsawa 1981] T. Ohsawa, "A remark on the completeness of the Bergman metric", Proc. Japan Acad. Ser. A Math. Sci. 57:4 (1981), 238-240. MR 82j:32053 Zbl 0508.32008

[Ramadanov 1967] I. Ramadanov, "Sur une propriété de la fonction de Bergman", C. R. Acad. Bulgare Sci. 20 (1967), 759-762. MR 37 \#1632 Zbl 0206.09002

[Wong 1977] B. Wong, "Characterization of the unit ball in $\mathbb{C}^{n}$ by its automorphism group", Invent. Math. 41:3 (1977), 253-257. MR 58 \#11521 Zbl 0385.32016 
Received April 17, 2012. Revised September 4, 2012.

ROBERT E. GREENE

DEPARTMENT OF MATHEMATICS

UNIVERSITY OF CALIFORNIA

Los ANGEles, CA 90095

UNITED STATES

greene@math.ucla.edu

KANG-TAE KIM

DEPARTMENT OF MATHEMATICS

PoHANG University of SCIENCE AND TECHNOLOGY

POHANG 790-784

SOUTH KOREA

kimkt@postech.ac.kr

STEVEN G. KRANTZ

DEPARTMENT OF MATHEMATICS

WASHINGTON UNIVERSITY

CAMPUS BoX 1146

SAINT LOUIS, MO 63130

UNITED STATES

sk@math.wustl.edu

AERYEONG SEO

SCHOOL OF MATHEMATICS

Korea Institute for AdVAnCED Study

SEOUL 151

SOUTH KOREA

inno827@postech.ac.kr 


\title{
PACIFIC JOURNAL OF MATHEMATICS
}

\author{
msp.org/pjm
}

Founded in 1951 by E. F. Beckenbach (1906-1982) and F. Wolf (1904-1989)

\section{EDITORS}

V. S. Varadarajan (Managing Editor)

Department of Mathematics

University of California

Los Angeles, CA 90095-1555

pacific@math.ucla.edu

Paul Balmer

Department of Mathematics

University of California

Los Angeles, CA 90095-1555

balmer@math.ucla.edu

Daryl Cooper

Department of Mathematics

University of California

Santa Barbara, CA 93106-3080 cooper@math.ucsb.edu

Jiang-Hua $\mathrm{Lu}$

Department of Mathematics

The University of Hong Kong

Pokfulam Rd., Hong Kong jhlu@maths.hku.hk
Don Blasius

Department of Mathematics University of California

Los Angeles, CA 90095-1555

blasius@math.ucla.edu

Robert Finn

Department of Mathematics Stanford University

Stanford, CA 94305-2125

finn@math.stanford.edu

Sorin Popa

Department of Mathematics

University of California

Los Angeles, CA 90095-1555

popa@math.ucla.edu

Paul Yang

Department of Mathematics

Princeton University

Princeton NJ 08544-1000

yang@math.princeton.edu

\section{PRODUCTION}

Silvio Levy, Scientific Editor, production@msp.org

\section{SUPPORTING INSTITUTIONS}

ACADEMIA SINICA, TAIPEI

CALIFORNIA INST. OF TECHNOLOGY

INST. DE MATEMÁTICA PURA E APLICADA

KEIO UNIVERSITY

MATH. SCIENCES RESEARCH INSTITUTE

NEW MEXICO STATE UNIV.

OREGON STATE UNIV.

\author{
STANFORD UNIVERSITY \\ UNIV. OF BRITISH COLUMBIA \\ UNIV. OF CALIFORNIA, BERKELEY \\ UNIV. OF CALIFORNIA, DAVIS \\ UNIV. OF CALIFORNIA, LOS ANGELES \\ UNIV. OF CALIFORNIA, RIVERSIDE \\ UNIV. OF CALIFORNIA, SAN DIEGO \\ UNIV. OF CALIF., SANTA BARBARA
}

\author{
Vyjayanthi Chari \\ Department of Mathematics \\ University of California \\ Riverside, CA 92521-0135 \\ chari@math.ucr.edu \\ Kefeng Liu \\ Department of Mathematics \\ University of California \\ Los Angeles, CA 90095-1555 \\ liu@math.ucla.edu \\ Jie Qing \\ Department of Mathematics \\ University of California \\ Santa Cruz, CA 95064 \\ qing@cats.ucsc.edu
}

These supporting institutions contribute to the cost of publication of this Journal, but they are not owners or publishers and have no responsibility for its contents or policies.

See inside back cover or msp.org/pjm for submission instructions.

The subscription price for 2013 is US \$400/year for the electronic version, and \$485/year for print and electronic.

Subscriptions, requests for back issues and changes of subscribers address should be sent to Pacific Journal of Mathematics, P.O. Box 4163, Berkeley, CA 94704-0163, U.S.A. The Pacific Journal of Mathematics is indexed by Mathematical Reviews, Zentralblatt MATH, PASCAL CNRS Index, Referativnyi Zhurnal, Current Mathematical Publications and the Science Citation Index.

The Pacific Journal of Mathematics (ISSN 0030-8730) at the University of California, c/o Department of Mathematics, 798 Evans Hall \#3840, Berkeley, CA 94720-3840, is published monthly except July and August. Periodical rate postage paid at Berkeley, CA 94704, and additional mailing offices. POSTMASTER: send address changes to Pacific Journal of Mathematics, P.O. Box 4163, Berkeley, CA 94704-0163.

PJM peer review and production are managed by EditFLOW ${ }^{\circledR}$ from Mathematical Sciences Publishers.

PUBLISHED BY

mathematical sciences publishers

nonprofit scientific publishing

http://msp.org/

(C) 2013 Mathematical Sciences Publishers 


\section{PACIFIC JOURNAL OF MATHEMATICS}

Volume $262 \quad$ No. $2 \quad$ April 2013

Certifying incompressibility of noninjective surfaces with scl

DANNY CALEGARI

Global well-posedness for the 3D rotating Navier-Stokes equations with highly oscillating initial data

QIONGLEI CHEN, CHANGXING MiAO and ZHIFEI ZHANG

Presenting Schur superalgebras

HOUSSEIN El TURKEY and JONATHAN R. KUJAWA

Classifying zeros of two-sided quaternionic polynomials and computing zeros of two-sided polynomials with complex coefficients

FENG LIANGGUI and ZHAO KAIMING

Coxeter groups, imaginary cones and dominance

XIANG FU

Semicontinuity of automorphism groups of strongly pseudoconvex domains: The low differentiability case

Robert E. Greene, Kang-Tae Kim, Steven G. Krantz and

AERYEONG SEO

Klein four-subgroups of Lie algebra automorphisms

JING-SONG HUANG and JUN YU

Fractal entropy of nonautonomous systems

Rui KuANG, WEN-ChiaO CHENG and BING LI

A GJMS construction for 2-tensors and the second variation of the total

$Q$-curvature

YoshiniKo MaTSUMOTO

Droplet condensation and isoperimetric towers

Matteo Novaga, Andrei Sobolevski and Eugene Stepanov

Brauer's height zero conjecture for metacyclic defect groups

BENJAMIN SAMBALE 